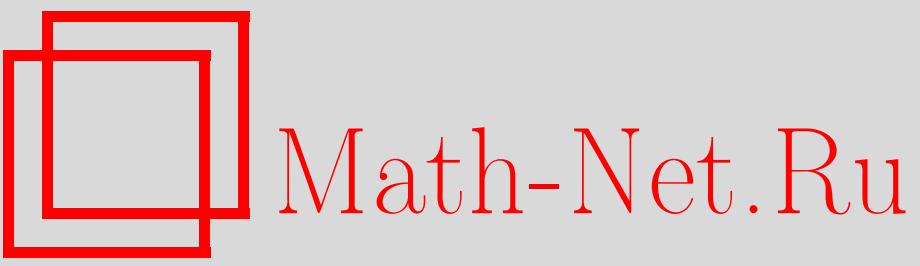

И. А. Дынников, С. П. Новиков, Топология квазипериодических функций на плоскости, УМH, 2005, том 60, выпуск 1, 3-28

DOI: https://doi.org/10.4213/rm1386

Использование Общероссийского математического портала Math-Net.Ru подразумевает, что вы прочитали и согласны с пользовательским соглашением

http://www.mathnet.ru/rus/agreement

Параметры загрузки:

IP: 44.207 .124 .84

26 апреля 2023 г., 12:35:18 


\title{
ТОПОЛОГИЯ КВАЗИПЕРИОДИЧЕСКИХ ФУНКЦИЙ НА ПЛОСКОСТИ
}

\author{
И. А. Дынников, С. П. Новиков
}

В настоящей работе излагается топологическая теория квазипериодических функций на плоскости. Начало развития этой теории было положено московской топологической группой (использовавшей иную терминологию) в начале $1980-\mathrm{x}$ годов. Это было мотивировано потребностями физики твердого тела, приведшими к необходимости изучения некоторого специального случая гамильтоновых слоений на поверхностях Ферми с многозначным гамильтонианом [1]. Их неожиданные топологические свойства, открытые в 1980-х [2], [3] и 1990-х [4]-[6], в итоге привели к нетривиальным физическим заключениям [7], [8] с помощью рассмотрения так назьваемого геометрического предела сильного магнитного поля [9]. Переформулировка задачи в терминах квазипериодических функций и обообщение на случай более высоких размерностей, сделанные в 1999 гг. [10], приводят к новому интересному подходу. Можно сказать, что для монокристаллического нормального металла, помещенного в магнитное поле, полуклассические траектории электронов в пространстве квазиимпульсов - это в точности линии уровня квазипериодической функции с тремя квазипериодами, которая является ограничением закона дисперсии на плоскость, перпендикулярную магнитному полю. Изучение топологических свойств уровней квазипериодических функций на плоскости с произвольным числом квазипериодов было начато в 1999 г., когда для случаев четырех квазипериодов были сформулированы некоторые новые идеи [10]. Последний раздел настоящей работы содержит полное доказательство этих резултатов, основанное на развитой в [11], [12] технике. Недавно были найдены некоторые новые физические приложения общей задачи [13].

Библиография: 29 названий

\section{СОДЕРЖАНИЕ}

1. Квазипериодические функции

2. Квазипериодические грушш в анализе, геометрии и теории динамических систем, возникающих в естественных науках .............

3. Топология и динамика квазипериодических функций на плоскости: случай трех квазипериодов. Электрическая проводимость в ме-

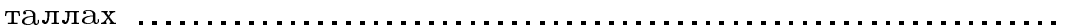

4. Устойчивая топологическая полная интегрируемость для $n=4$ квазипериодов

Список литературы . . . . . . . . . . .

Работа первого автора выполнена при частичной поддержке РФФИ (грант № 02-01-00659); работа второго автора выполнена при частичной поддержке Президиума РАН (программа "Математические методы нелинейной динамики"). 


\section{1. Квазипериодические функции}

Обозначим через $\mathbb{T}^{n}=\mathbb{R}^{n} / \mathbb{Z}^{n}$ тор размерности $n$, через $\nu: \mathbb{R}^{n} \rightarrow \mathbb{T}^{n}=\mathbb{R}^{n} / \mathbb{Z}^{n}$ стандартную проекцию.

Мы будем говорить, что гладкая вешественная функция $\varphi(y)=\varphi\left(y^{1}, \ldots, y^{k}\right)$ на $k$-мерной плоскости $\mathbb{R}^{k}$ квазипериодична с $n$ квазипериодами (частотами), если ее можно представить в виде композиции $\varphi(y)=f(x(y))$ :

$$
\varphi=f \circ \nu \circ \iota
$$

где $\iota: \mathbb{R}^{k} \rightarrow \mathbb{R}^{n}-$ некоторое аффинное вложение:

$$
x^{s}=a_{r}^{s} y^{r}+x_{0}^{s}
$$

$f=f(x): \mathbb{T}^{n} \rightarrow \mathbb{R}$ - гладкая функция, причем $n \geqslant 2-$ минимально возможное целое, для которого существуют такие функция $f$ и аффинное вложение $\iota$. Здесь $s=1, \ldots, n$ и $r=1, \ldots, k$. В теории квазикристаллов пространство $\mathbb{R}^{k}$ (где $k=2,3$ ) принято называть физическим пространством, $\mathbb{R}^{n}$ - суперпространством. Каждая $n$-периодическая функция $f(x)$ порождает семейство потомков, получаемых варьированием начального вектора $x_{0}=\left(x_{0}^{1}, \ldots, x_{0}^{n}\right)$ в суперпространстве $\mathbb{R}^{n}$. Про любые два потомка $\varphi_{1}(y), \varphi_{2}(y)$ одной и той же функции $f$ говорят, что они родственные. Они имеют одни и те же частоты и обладают следующим свойством: для любого $\varepsilon>0$ найдется такой сдвиг $y \mapsto y+a$ физического пространства, что после него функция $\varphi_{2}(y)$ будет $\varepsilon$-близка к $\varphi_{1}(y),\left|\varphi_{2}(y+a)-\varphi(y)\right|<\varepsilon \forall y \in \mathbb{R}^{k}$.

Любая линейная функция $\lambda: \mathbb{R}^{k} \rightarrow \mathbb{R}$ вида $\lambda(y)=\ell(x(y))$ (или $\left.\lambda=\ell \circ \iota\right)$, где линейная функция $\ell: \mathbb{R}^{n} \rightarrow \mathbb{R}$ лежит в двойственной (взаимной) решетке $\left(\mathbb{Z}^{n}\right)^{*}$, т.е. такова, что $\ell\left(\mathbb{Z}^{n}\right) \subset \mathbb{Z}$, назьвается частотой функции $\varphi$. Множество частот квазипериодической функции образует свободную абелеву группу с $n$ естественными обрузуюшими $\lambda_{1}=\ell^{1} \circ \iota, \ldots, \lambda_{1}=\ell^{n} \circ \iota$, где функции $\ell^{s}(x)=x^{s}, s=1, \ldots, n$, образуют двойственный базис к базису периодов. Эту групу мы назьваем группой частот. Она является свободной абелевой подгруппой $\Gamma^{*}$ векторного пространства $\mathbb{R}^{*}$. Она одна и та же для всего семейства родственных квазипериодических функций (потомков одной $n$-периодической функции $f$ ).

Аналитически любую $n$-периодическую функцию можно представить в виде тригонометрического ряда

$$
f(x)=\sum_{\ell \in\left(\mathbb{Z}^{n}\right)^{*}} c_{\ell} \exp (2 \pi i \ell(x)) .
$$

Следовательно, любую квазипериодическую функцию можно представить подобным образом:

$$
\begin{aligned}
\varphi(y) & =\sum_{\lambda \in \Gamma^{*}} b_{\lambda} \exp (2 \pi i \lambda(y)) \\
& =\sum_{m} b_{m} \exp \left(2 \pi i \sum_{s=1}^{n} m_{s} \lambda_{s}(y)\right),
\end{aligned}
$$

где $m=\left(m_{1}, \ldots, m_{n}\right) \in \mathbb{Z}^{n}$. По определению множество базисных частот $\lambda_{s}$ порождает пространство $\mathbb{R}^{k}$ над полем $\mathbb{R}$. 
В случае евклидова пространства $\mathbb{R}^{k}$ имеется естественное отождествление $\mathbb{R}^{k}=\left(\mathbb{R}^{k}\right)^{*}$, поэтому группу частот можно рассматривать какподгрупшу $\Gamma=\Gamma^{*} \subset \mathbb{R}^{k}$ в физическом пространстве $\mathbb{R}^{k}$.

С каждым семейством родственных квазипериодических функций ассоциируется группа аффинных симметрий: по определению эта группа $\widetilde{G}$ состоит из таких аффинных преобразований

$$
g: \mathbb{R}^{k} \rightarrow \mathbb{R}^{k}
$$

физического пространства, что

$$
g\left(\Gamma^{*}\right) \subset \Gamma^{*}
$$

где $\Gamma^{*}$ рассматривается как подмножество группы сдвигов: $\Gamma^{*} \subset \mathbb{R}^{k}$. В случае евклидова пространства $\mathbb{R}^{k}$ мы вводим группу симметрий $G \subset \widetilde{G}$, которая состоит только из изометрий $g$, для которых $g\left(\Gamma^{*}\right)=\Gamma^{*}$.

Эта группа подходит под общее определение квазикристаллографической групшы, введенное С.П. Новиковьм и А. П. Веселовым в 1980-х для того, чтобы дать ответ на вопрос: какова симметрия квазикристаллов (см. [14])? Согласно этому определению, пересечение $G \cap \mathbb{R}^{k} \subset \mathrm{Iso}\left(\mathbb{R}^{k}\right)$ кристаллографической групшы с подгруппой трансляций должно быть свободной конечно порожденной абелевой группой. В нашем случае это в точности група $\Gamma^{*}$. Наше определение допускает бесконечную фактор-групу "вращений” $G /\left(G \cap \mathbb{R}^{k}\right) \subset O_{k}$. С. В. Пиунихин изучал эти группы при $k=2,3$ в серии работ (см. [14]).

ПримеР 1. Рассмотрим двумерньй случай, $k=2$. Пусть $\theta$ - некоторое единичное по абсолютной величине комплексное число, $|\theta|=1, \theta=\exp (i \psi)$, удовлетворяющее следующему уравнению:

$$
P(\theta)=\theta^{n}+a_{1} \theta^{n-1}+\cdots+a_{n-1} \theta+1=0,
$$

в котором все коэффициенты целые, $a_{s} \in \mathbb{Z}$, и, кроме того, $a_{s}=a_{n-s}$. Комплексные числа (вешественные 2-векторы) $\lambda_{1}=1, \lambda_{2}=\theta, \ldots, \lambda_{n}=\theta^{n-1}$ порождают группу частот $\Gamma^{*} \subset \mathbb{C}=\mathbb{R}^{2}$ с нетривиальной группой вращательных симметрий $g \rightarrow g \exp (i \psi)$. Нетрудно подобрать многочлен $P$ с корнем в точке вида $\theta=\exp (i \psi)$ так, чтобы отношение $\psi /(2 \pi)$ было иррационально.

Очень сложные примеры квазикристаллографических груп имеются в случае $k=3$ (см. [14]).

\section{2. Квазипериодические группы в анализе, геометрии и теории динамических систем, возникающих в естественных науках}

2.1. Квазипериодические функции на вещественной прямой. Рассмотрим случай $k=1$. В XIX веке одномерные квазипериодические функции с $n$ квазипериодами появлялись в теории вполне интегрируемых гамильтоновых систем классической механики с $n$ степенями свободы. Согласно так назьваемой теореме Лиувилля, их интегрируемость следует из существования $n$ штук гладких независимых попарно коммутирующих интегралов движения. Если их совместный уровень компактен, то для лежашей на нем траектории временна́я зависимость пространственных координат $x^{r}(t)$ будет квазипериодической функцией с $n$ квазипериодами. Поэтому изучение 
возмущений вполне интегрируемых систем нужно начинать с рассмотрения невозмущенных квазипериодических решений. В этой области имеется цельй ряд фундаментальных работ (см. [15], [16]).

2.2. Квазипериодические функции в теории нелинейных уравнений с частными производными. Вполне интегрируемые системы УрЧП теории солитонов приводят к квазипериодическим функциям с $k>1$. Среди них такие очень известные $(1+1)$-системы, как $\mathrm{K} д \Phi\left(u_{t}=6 u u_{x}\right)$ и синус-Гордон $\left(u_{t t}=\right.$ $\left.u_{x x}+\sin \{u(x, t)\}\right)$, интегрируемые так назьваемым методом обратной задачи рассеяния для быстро убывающих начальных значений. В 1970-х был открыт счетный набор непрерьвных семейств точных гладких вещественных "конечнозонных" решений этих уравнений (см. [15], [16]). Эти решения квазипериодичны по $x, t$ и зависят от набора параметров $a, a^{\prime}$ :

$$
u(x, t)=F\left(x U+V t+U_{0} ; a\right)
$$

для $\mathrm{K}$ д и

$$
\exp \{i u(x, t)\}=F^{\prime}\left(U^{\prime} x+V^{\prime} t+U_{0}^{\prime} ; a^{\prime}\right)
$$

для синус-Гордон. Здесь в обоих случаях $u(x, t)$ вешественно, $F, F^{\prime}$ - гладкие $n$-периодическая функции от $n$ переменных (т.е. гладкие функции на $n$-мерном торе). Их можно выразить через специальные функции, а именно тета-функции гиперэллиптических римановых поверхностей рода $n$. В формулах вьше $U, U^{\prime}, V, V^{\prime}$ - это $n$-векторы периодов некоторых абелевых дифференциалов второго рода (см. [3]). Заметим, что для системы синус-Гордон функция $u=(1 / i) \log F^{\prime}$ в случае общего положения является многозначной функцией на "вешественном" $n$-мерном торе, вложенном в комплексный $2 n$-мерньй тор Якоби, ассоциированньй с комплексной гиперэллиптической римановой поверхностью. В этом случае мы имеем $k=2$. Для знаменитых вполне интегрируемых $(2+1)$-систем (скажем, для КП и других) мы приходим к квазипериодическим решениям вида $u(x, y, t)$, которые являются квазипериодическими функциями от $k=3$ физических переменных. При изучении зависимости этих решений от так назьваемых высших времен могут получаться произвольные значения $k$.

2.3. Квазипериодические функции и квазикристаллы. Совсем другого рода примеры происходят из физики твердого тела. В 1980-х годах были открыты новые виды двумерных и трехмерных материалов, которые были названы квазикристаллами. Оптический анализ расположения атомов указывал на наличие группы частот, несовместимой с обычной кристаллической структурой. Например, при $k=2$ наблюдаемая группа частот $\Gamma^{*}$ могла порождаться корнями пятой степени из единишы:

$$
\lambda_{r}=\eta^{r} \in \Gamma^{*}, \quad r=0,1,2,3, \quad \eta^{5}=1, \quad P(\eta)=0
$$

где

$$
P(z)=z^{4}+z^{3}+z^{2}+z+1 .
$$

Напомним: наше расширение идеи симметрии (см. выше) допускает врашательную симметрию даже бесконечного порядка. 
Имеются две математические модели квазикристаллов. Будем считать атомы точками, образующими дискретное подмножество $\left\{x_{A}\right\}$ в физическом пространстве $\mathbb{R}^{k}$ и расположенными так, что

(а) найдется пара положительных "радиусов" $\rho_{1}>\rho_{2}>0$ со свойством: $\left|x_{A}-x_{A^{\prime}}\right| \geqslant \rho_{2}$ для всех пар $A, A^{\prime}, A \neq A^{\prime}$

(б) для любой точки $x \in \mathbb{R}^{k}$ найдется такая точка $x_{A}$, что $\left|x_{A}-x\right| \leqslant \rho_{1}$.

Мы назьваем такое множество точек квазипериодическим, если распределение $\sum_{A} \delta\left(x-x_{A}\right)$ можно разложить в ряд Фурье с конечно порожденной свободной абелевой группой частот $\Gamma^{*}$.

Согласно другой модели, физическое пространство $\mathbb{R}^{k}$ должно представляться в виде “квазипериодического покрытия”. Это означает следующее.

(a) Пространство покрыто счетным числом многогранников $P_{B}, \mathbb{R}^{k}=\bigcup_{B} P_{B}$, где пересечение $P_{B} \bigcap P_{B^{\prime}}$ любых двух $B, B^{\prime}$ является гранью.

(б) $\mathrm{C}$ точностью до сдвига среди них имеется лишш конечное число различных многогранников $P_{1}, \ldots, P_{q}$.

(в) Ассоциируем с каждым многогранником $P_{q}$ некоторую константу $c_{q}$ и рассмотрим функцию, которая равна $c_{j}$ внутри каждого многогранника $P_{B}$, полученного сдвигом из $P_{j}$. Мы получим кусочно постоянную функцию $c(x)$ от $x \in \mathbb{R}^{k}$, определяемую нашим покрытием и выбором констант $c_{j}$. Покрытие назьвается квазипериодическим, если для любого выбора констант $c_{j}$ функция $c(x)$ квазипериодична, т.е. представляется в виде тригонометрического ряда с конечно порожденной свободной абелевой группой частот $\Gamma^{*}$.

Имеется знаменитое покрытие плоскости $\mathbb{R}^{2}$ ромбами двух типов: один с углами $\pi / 5$ и $4 \pi / 5$, другой $-2 \pi / 5$ и $3 \pi / 5$. Оно назьвается “покрытием Пенроуза". Это покрытие квазипериодично, что было открыто через несколько лет после первоначальной работы Пенроуза (историю вопроса и подробности см. в [14]). Для объяснения роста квазикристаллов в терминах покрытий физиками и математиками была разработана замечательная теория “локальных правил". В этой модели атомы располагаются в вершинах элементов покрытия.

Обе модели можно получить из следующей конструкции. Пусть нам дана "суперрешетка" Г полного ранга в суперпространстве $\mathbb{R}^{n}$, которое разложено в прямую сумму $\mathbb{R}^{n}=\mathbb{R}^{k} \oplus \mathbb{R}^{n-k}$, где $\mathbb{R}^{k}$ - наше физическое пространство. Пусть $p: \mathbb{R}^{n} \rightarrow \mathbb{R}^{k}$ и $q: \mathbb{R}^{n} \rightarrow \mathbb{R}^{n-k}$-естественные проекции. Зафиксируем некоторый конечный $n$-полиэдр $D \subset \mathbb{R}^{n-k}$ и рассмотрим “ $D$-трубчатую окрестность" $D_{q}=q^{-1}(D) \subset \mathbb{R}^{n}$ физического подпространства $\mathbb{R}^{k} \subset \mathbb{R}^{n}$. Предположим, что граница полиэдра $D$ не пересекается с $q(\Gamma)$, или, эквивалентньм образом, $\partial D_{q} \cap \Gamma=\varnothing$. Тогда множество точек

$$
p\left(\Gamma \cap D_{q}\right) \subset \mathbb{R}^{k}
$$

квазипериодично в смысле данного вьше определения.

Разбив $\mathbb{R}^{n}$ специальньм образом на многогранники, исходя из решетки Г и полиэдра $D$, мы получим квазипериодическое покрытие пространства $\mathbb{R}^{k}$, в котором элементы покрытия - это пересечения подпространства $\mathbb{R}^{k}$ с $n$-клетками разбиения (см. об3ор [14]).

Очень интересные примеры нетривиальных групп симметрий появляются в случае суперпространства $\mathbb{R}^{4}$, оснашенного метрикой Минковского и такой решеткой $\Gamma \cong \mathbb{Z}^{4}$, 
что физическое подпространство $\mathbb{R}^{2}$, являюшееся пространственноподобным (т.е. евклидовым), инвариантно относительно некоторого отображения из групшы $O(3,1)$.

Подпространства $\mathbb{R}^{l, m}$ с $l+m=n$ также могут появляться в интересных случаях.

2.4. Квазипериодические функции в теории проводимости. Теперь мы опишем ситуацию, дающую основную мотивировку нашей топологической и динамической теории.

Для любого монокристаллического металла в физическом пространстве $\mathbb{R}^{3}$ имеется решетка Г. Однако наши геометрические построения будут относиться совсем к другому пространству, а именно трехмерному тору квазиимпульсов $\mathbb{T}^{3}$, являющемуся факторпространством двойственного трехмерного пространства $\left(\mathbb{R}^{3}\right)^{*} \cong \mathbb{R}^{3}$ по двойственной (взаимной) решетке $\Gamma^{*}$. "Блоховские" состояния квантовых электронов параметризуются парами $(m, p)$, в которых $p$ является точкой пространства квазиимпульсов $p \in \mathbb{T}^{3}=\mathbb{R}^{3} / \Gamma^{*}$, а $m$ - натуральное число, нумерующее ветвь закона дисперсии $f(p)=\epsilon_{m}(p): \mathbb{T}^{3} \rightarrow \mathbb{R}$. В дальнейшем мы всегда будем рассматривать только одну ветвь, поэтому мы будем пропускать индекс $m$ в обозначениях. Мы будем считать, что $f(p)=\epsilon(p)$ - функция Морса на трехмерном торе, или, другими словами, 3 -периодическая функщия Морса на накрьвающем евклидовом пространстве $\mathbb{R}^{3}$. При нулевой температуре электроны заполняют "море Дирака" $\epsilon(p) \leqslant \epsilon_{F}$, где "энергия Ферми" $\epsilon_{F}$ зависит от числа свободных электронов в металле. Мы предполагаем, что $\epsilon_{F}$ является регулярным значением функции Морса $f=\epsilon(p)$. При низкой температуре мы имеем дело только с “возбужденными” электронами вблизи уровня Ферми $\epsilon(p)=\epsilon_{F}$.

Геометрически уровень Ферми представляет собой двумерную поверхность $M_{F} \subset \mathbb{T}^{3}$ в пространстве квазиимпульсов. Эта поверхность неособа и гомологична нулю в трехмерном торе. Предположим, что она связна.

Топологический ранг $r\left(M_{F}\right)$ поверхности Ферми определяется как ранг образа первой группы гомологий поверхности $M_{F}$ при отображении $i_{*}: H_{1}\left(M_{F}, \mathbb{Z}\right) \rightarrow$ $H_{1}\left(\mathbb{T}^{3}, \mathbb{Z}\right) \cong \mathbb{Z}^{3}$, индуцированном включением $i: M_{F} \hookrightarrow \mathbb{T}^{3}$. Так как $i_{*}\left(H_{1}\left(M_{F}\right)\right)$ образует подрешетку в $\mathbb{Z}^{3}$, мы во всех случаях имеем $r \in\{0,1,2,3\}$.

Для лития, например, топологический ранг поверхности Ферми равен нулю (в этом случае поверхность Ферми представляет собой топологическую двумерную сферу), в то время как этот ранг равен трем для меди, золота, платины и некоторых других драгоценных металлов. Род Ферми-поверхности золота, например, равен четырем.

Нам особо интересен случай, когда топологический ранг поверхности Ферми равен трем, т.е. максимально возможньй. В этом случае род поверхности не может быть меньше трех. Действительно, так как поверхность гомологична нулю в трехмерном торе, образ $\iota^{*}\left(H^{1}\left(\mathbb{T}^{3}\right), \mathbb{R}\right)=\mathbb{R}^{3} \subset H^{1}\left(M_{F}, \mathbb{R}\right)$ является лагранжевым подпространством в симплектическом пространстве $H^{1}\left(M_{F}, \mathbb{R}\right)$. Последнее означает, что $\operatorname{rank}\left(H^{1}\left(M_{F}\right)\right) \geqslant 6$, откуда следует, что род поверхности $M_{F}$ не меньше трех.

Интересные динамические явления происходят в присутствии магнитного поля. В полуклассическом приближении электрон, рассматриваемьй как точка в пространстве квазиимпульсов, движется вдоль линии постоянной энергии в плоскости $\mathbb{R}_{B, p_{0}}^{2}$, перпендикулярной магнитному полю $B$ и проходяшей через начальное положение $p_{0}$ квазиимпульса. Можно сказать, что это гамильтонова система на трехмерном торе квазиимпульсов со скобкой Пуассона

$$
\left\{p_{j}, p_{l}\right\}=\frac{e}{c} B_{j l}=\frac{e}{c} \varepsilon_{j l q} B^{q}
$$


и гамильтонианом $f=\epsilon(p)$ :

$$
\frac{d p_{j}}{d t}=\left\{p_{j}, \epsilon(p)\right\}
$$

так что при движении сохраняются энергия и линейный казимир скобки Пуассона, т.е. направление плоскостей, ортогональных магнитному полю. Траектории можно рассматривать как слои гамильтонова слоения $\omega=0$ на поверхности Ферми, заданного замкнутой 1-формой $\omega=\left.\sum_{j} B^{j} d p_{j}\right|_{M_{F}}$.

Согласно принципу "предела сильного магнитного поля", выработанному в начале 1960-х годов И.М. Лифшицом, М.Я. Азбелем, М. И. Кагановьм и В.Г. Песчанским, все основные свойства электрической проводимости в присутствии однородного магнитного поля $B$ разумной силы должны вытекать из структуры описанной вьше динамической системы на поверхности Ферми (см. [9], [17]-[19]). Для обычных нормальных металлов (например, таких, как золото) это приближение можно использовать для довольно сильных с практической точки зрения магнитных полей (порядка 1 Tesla < $|B|<10^{3}$ Tesla для низкой температуры; напомним, что 1 Tesla $=10^{4}$ Gauss). Если магнитное поле слишком сильное, то полуклассическое приближение перестает быть применимым. Если магнитное поле слишком слабое, то дрейф электронного квазиимпульса также будет медленным и расстояние, пройденное квазиимпульсом за время свободного пробега электрона, станет недостаточньм для наблюдаемого изменения проводимости.

Однако в 1960-х годах исследование описанной динамической системы было лишш начато. В [20] была сделана некоторая концептуальная ошибка, после чего дальнейшие исследования остановились и возобновились лишш много лет спустя в работах [1]-[8], [11], [12], [21]-[24].

Для нас принципиально важно следующее:

траектории электрона совпадают со связньми компонентами линий уровня $\epsilon(p)=\epsilon_{F}$ функции $\epsilon$, ограниченной на плоскости, ортогональные магнитному полю $B$; иначе говоря, эти траектории являются связными компонентами линий уровня функций, которые образуют семейство родственных квазипериодических функций с тремя квазипериодами.

В работе [10] было начато обобщение этого исследования на случай большего числа квазипериодов. В частности, были сформулированы некоторые идеи и результаты для случая $n=4$. Настояшая работа содержит первое полное доказательство тех утверждений (надлежашим образом исправленных), основанное на технике, развитой в $[11],[12]$.

Пусть на $n$-мерном торе задана постоянная скобка Пуассона $B_{j k}$ ранга два. Тогда любой гамильтониан $f(p)=\epsilon(p): \mathbb{T}^{n} \rightarrow \mathbb{R}$ задает гамильтонову систему, траектории которой в точности совпадают с линиями уровня $\epsilon(p)=$ const ограничения функщии $\epsilon$ на плоскости $\mathbb{R}_{B, a}^{2}$, определенные следующим образом. Имеется ровно $n-2$ независимых линейных казимиров $K_{1}, \ldots, K_{n-2}$, где $K_{j}(p)=K_{j}^{l} p_{l}$ таких, что $\left\{p_{s}, K_{j}\right\}=0=K_{j}^{l} B_{s l}$. Мы полагаем

$$
\mathbb{R}_{B, a}^{2}=\left\{K_{1}=a_{1}, \ldots, K_{n-2}=a_{n-2}\right\},
$$


где $a=\left(a_{1}, \ldots, a_{n-2}\right)$. Так что в качестве наших траекторий выступают линии уровня квазипериодических функций, образуюших семейство потомков одной и той же $n$-периодической функции $\epsilon(p)$, на семействе параллельных двумерных плоскостей $\mathbb{R}_{B, a}^{2}$. Они зависят от констант $a_{1}, \ldots, a_{n-2}$. боте.

Исследование топологии этой задачи занимает центральное место в настояшей ра-

Современные экспериментальные технологии позволяют строить поверхности с целым рядом предписанных малых флуктуаций. В частности, можно получать квазипериодические конструкции с произвольньм числом квазипериодов. Это даст нам слабый двумерный квазипериодический электрический потенциал $V(x, y)$. В сильном магнитном поле $B$ электроны движутся по циклотронным орбитам, возмущенным потенциалом $V$. Усреднение потенциала по циклотронным орбитам на уровне Ферми дает “эффективньй” потеншиал $V_{B}^{\mathrm{eff}}$, отвечающий за дрейф электронных орбит, которьй происходит вдоль линии уровня $V_{B}^{\mathrm{eff}}(x, y)=$ const. При этом эффективный потенциал $V_{B}^{\mathrm{eff}}(x, y)$ имеет те же квазипериоды, что и $V(x, y)$.

Эти исследования, экспериментальное и теоретическое, первоначально были проведены для периодических потенциалов только с $n \leqslant 2$ периодами (см. [25]), но в работе [13] отмечено, что их можно распространить и на квазипериодические потенциалы; для квазипериодических случаев трех и четырех квазипериодов были сделаны новые предсказания, основанные на топологических результатах, полученных в серии работ (см. [10], [12], [24]).

\section{3. Топология и динамика квазипериодических функций на плоскости: случай трех квазипериодов. Электрическая проводимость в металлах}

Зададим следующий общий вопрос. Как могут вьглядеть линии уровня $\varphi=$ const квазипериодической функции $\varphi$ на плоскости с $n$ квазипериодами? Как задача топологии и динамических систем этот вопрос был сформулирован для случая трех квазипериодов в начале 1980-х годов в работе [1]. В случае обшего положения интересующая нас линия уровня представляет собой одномерное подмногообразие в $\mathbb{R}^{2}$, т.е. объединение кривых. Мы будем назьвать эти кривые траекториями, поскольку в первоначальной постановке задачи они появились как полуклассические траектории электрона в магнитном поле на поверхности Ферми. Некоторые из этих кривых могут быть замкнуты в $\mathbb{R}^{2}$ (компактны), а другие незамкнуты в $\mathbb{R}^{2}$ (открыты). Поставим следующие вопросы.

Вопрос 1. Верно ли, что размер всех компактных траекторий равномерно ограничен (для фиксированного уровня функции $\varphi$ )?

Вопрос 2. Имеют ли открытые траектории какое-либо хорошее асимптотическое поведение на бесконечности?

Первые результаты были получены в работе [2]. Во второй половине 1980-х стало ясно, что наш вопрос 2 правильно формулировать следуюшим образом: имеет ли каждая открытая траектория "сильное асимптотическое направление" в $\mathbb{R}^{2}$, т.е. лежит в полосе конечной ширины и проходит ее “от $-\infty$ до $+\infty$ ”? Это уточнение задачи было сделано в статье [3]. В работе [4] результаты работы [2] были улучшены в соответствии с новой формулировкой задачи. Важное продвижение было сделано в работе [6], но в течение длительного времени не было развито никаких приложений. Физические приложения были найдены позже в работах [7], [8]. 
В физически важном случае $n=3$ положительньй ответ на наш вопрос 1 легко следует из довольно элементарного рассуждения. Для $n>3$ этот вопрос сложнее и обсуждается ниже. Вопрос 2 чрезвычайно нетривиален уже для $n=3$. Как упомянуто вьше, задача изучения асимптотического поведения открытых траекторий электронов, т.е. открытых связных компонент линии уровня квазипериодической функции с тремя квазипериодами, изучалась в [2], [4], [6]. После работы [6] стало окончательно ясно, что для семейства родственных квазипериодических функций, соответствующего "типичному" направлению магнитного поля (которое рассматривается как направление некоторой плоскости $\mathbb{R}^{2} \subset \mathbb{R}^{3}$ ), любая линия уровня либо вовсе не имеет открытых регулярных компонент, либо все такие компоненты имеют сильное асимптотическое направление. Последнее означает, что каждая открытая кривая имеет такую параметризацию $\gamma(t)$, что для некоторого ненулевого вектора $\left(x_{1}, y_{1}\right)$ вьполнено следующее:

$$
\gamma(t)=\left(x_{0}, y_{0}\right)+t \cdot\left(x_{1}, y_{1}\right)+O(1)
$$

Ниже мы укажем точный смысл слов “типичное направление” и дадим ссылки на работы, содержащие доказательства соответствующих результатов. В первой работе [2], завершенной в заметке [4], подобные результаты были получены для специального случая малых возмущений магнитных полей, имеющих "рациональное" направление. Мы вновь обратимся к этому специальному случаю в следующем разделе, где обсуждаются квазипериодические функции с четырьмя квазипериодами.

В работах [7], [8] представлены приложения этих исследований к объяснению электрической проводимости в сильном магнитном поле. Они основаны на результатах школы Лифшица 1960-х. Физики вычислили вклад индивидуальных траекторий простого вида в тензор проводимости. Эти вычисления вошли в классические учебники (см. [9], [17]). В случае, когда все тректории компактны, компоненты тензора проводимости, ортогональные магнитному полю $B$, убьвают со скоростью $|B|^{-1}$ или $|B|^{-2}$ при росте $|B|$ в предположении, что направление $B$ фиксировано. В то же время были найдены некоторые специальные примеры открытых траекторий, лежащих в полосах конечной ширины, и вычислен их вклад в проводимость. Как отмечено в [7], [8], упомянутое выгисление легко распространить на случай обших траекторий того же вида. Проекция вклада такой траектории в тензор проводимости на плоскость, ортогональную $B$, имеет два собственных значения, одно из которых нулевое, а другое ненулевое. Так как вклад замкнутых траекторий при росте $|B|$ стремится к нулю, при достаточно сильном поле $B$ наблюдаемый тензор проводимости будет зависеть только от открытых траекторий.

Тем не менее, наблюдаемьй физический тензор проводимости образуется как сумма вкладов всех электронных траекторий. Что можно сказать об этом тензоре из качественного описания нашей системы, определенной на квантовом уровне?

Для получения нетривиального физического результата необходимо несколько больше того, что явно сфформулировано в теоремах работы [6]. Но к счастью, наша динамическая система обладает также некоторьм важньм свойством, которое легко извлекается из доказательств результатов в [2], [6]. Это свойство, которое мы называем топологическим резонансом, влечет следуюшее для поведения открытых траекторий: 
Для “типичного” семейства $\left\{\varphi_{a}\right\}$ родственных квазипериодических функций $\varphi_{a}(p)=\left.\epsilon(p)\right|_{\mathbb{R}_{B, a}^{2}}$ сильное асимптотическое направление $\eta_{B}$ одинаково для всех некомпактных траекторий. Кроме того, имеется целочисленная двумерная плоскость $\mu \subset \mathbb{R}^{3}$ (т.е. плоскость, порожденная парой векторов двойственной решетки, $\mu \cap \mathbb{Z}^{3} \cong \mathbb{Z}^{2}$ ) такая, что $\eta_{B}$ есть направление пересечения плоскости $\mu$ с плоскостью, ортогональной магнитному полю:

$$
\eta_{B} \in \mu \cap \mathbb{R}_{B}^{2}
$$

Эта целочисленная плоскость $\mu$ локально жестка, т.е. не изменяется при малых шевелениях направления магнитного поля.

Свойство топологического резонанса нашей системы дает возможность получить серьезные приложения. Оно не было замечено в работах физиков, а в работе [20] была сделана концептуальная ошибка, которая привела к результатам, противоречащим топологическому резонансу. Эта ошибка была исправлена лишш в работах [7], [8], [22].

Для достаточно сильного $B$ направление $\eta_{B}$ является собственным, отвечающим нулевому собственному значению, направлением проекции тензора проводимости на плоскость, ортогональную магнитному полю. Целочисленную плоскость $\Pi \subset \mathbb{R}^{3}$ можно найти экспериментально, измерив собственный вектор $\eta_{B}$ для двух или более близких по направлению и достаточно сильных магнитных полей $B$. Мы отсылаем читателя к недавней работе [22] за более подробньм обсуждением физических аспектов задачи.

Опишем топологические свойства нашей задачи. Рассмотрим соответствуюшие объекты в накрываюшем трехмерном пространстве $\mathbb{R}^{3}$, в котором лежат дуальная решетка $\Gamma^{*}=\mathbb{Z}^{3} \subset \mathbb{R}^{3}$ и 3 -периодическая поверхность Ферми

$$
\widehat{M_{F}}=\nu^{-1}\left(M_{F}\right) \subset \mathbb{R}^{3},
$$

накрьвающая компактную

$$
M_{F} \subset \mathbb{T}^{3}
$$

(Напомним, что $\nu$ обозначает стандартную проекцию $\mathbb{R}^{3} \rightarrow \mathbb{T}^{3}$.) Электронные траектории в накрываюшем пространстве являются связньми компонентами пересечений 3-периодической поверхности Ферми с плоскостями, ортогональньми полю $B$. Обозначим через $M_{0}(B)$ топологическое замыкание объединения всех компактных траекторий, а через $L(B)$ - замыкание оставшейся части поверхности Ферми:

$$
L(B)=\overline{M_{F} \backslash M_{0}(B)} \text {. }
$$

Обозначим через $L_{l}(B)$ связные компоненты $L(B)$. В типичном случае $L(B)$ является компактной поверхностью с краем

$$
\partial L(B)=\bigcup_{l, s} \beta_{l s}
$$

где

$$
\partial L_{l}(B)=\bigcup_{s} \beta_{l s}
$$


Все граничные кривые $\beta_{s l}$ являются сепаратрисными циклами, причем в типичном случае можно считать, что каждый такой цикл $\beta_{s l}$ соединяет некоторое седло с самим собой, поскольку все остальные ситуации имеют положительную коразмерность в соответствующем функциональном пространстве. (В частности, мы предполагаем, что: между компонентами поля $B$ нет рациональной линейной зависимости $B$; рассматриваемое нами гамильтоново слоение, заданное уравнением $\omega=\left.\sum B^{i} d p_{i}\right|_{M_{F}}=0$, имеет только морсовские особенности; два различных седла не соединены сепаратрисой.)

Часть $M_{0}$ поверхности Ферми представляет собой объединение "цилиндров" $Z_{q}$, $M_{0}=\bigcup_{q} Z_{q}$, внутренность которых состоит из регулярных компактных траекторий, а "основаниями" являются либо сепаратрисные циклы, либо изолированные особые точки (центры). Число таких цилиндров конечно, и их объединение очивидным образом компактно. Отсюда немедленно следует положительный ответ на наш вопрос 1, сформулированньй в начале этого раздела:

размер всех компактных траекторий равномерно ограничен.

Мы назьваем части $L_{l}$ поверхности Ферми носителями открытых траекторий. По построению любая открытая траектория (рассматриваемая в $\mathbb{T}^{3}$ ) содержится в одном из таких носителей и (в случае общего положения) всюду плотна в нем. Пусть $D_{l s}^{2} \subset \mathbb{R}_{B, a}^{2}-$ двумерные диски, ортогональные магнитному полю, имеющие своим краем упомянутые вьше сепаратрисные циклы: $\partial D_{l s}^{2}=\beta_{l s}$. Мы определяем "замыкание" $N_{l}$ каждого носителя $L_{l}$ следуюшим образом:

$$
N_{l}=L_{l} \cup\left(\bigcup_{s} D_{l s}^{2}\right) .
$$

По построению мы имеем

$$
N_{l} \cap N_{l^{\prime}}=\varnothing
$$

при $l \neq l^{\prime}$.

Назовем нашу систему устойчивой топологически вполне интегрируемой, если род каждой поверхности $N_{l}$ равен единице и эта картина устойчива при достаточно малых возмущениях магнитного поля. Эргодические свойства гамильтоновых систем на двумерном торе изучались в работах [26], [27].

Мы назьваем нашу систему хаотичной, если род какого-либо $N_{l}$ больше единищы. Согласно основной теореме работы [6], такая ситуация всегда топологически нестабильна.

Согласно [7], [8], [22], для физических приложений принципиально важно следующее свойство топологического резонанса нашей системы. В устойчивом топологически вполне интегрируемом случае замыкания $N_{l}$ всех носителей открытых траекторий имеют один и тот же с точностью до знака класс гомологий:

$$
\left[N_{l}\right]= \pm \mu \in H_{2}\left(\mathbb{T}^{3}, \mathbb{Z}\right), \quad \mu \neq 0,
$$

которьй является неделимьм элементом группы $H_{2}\left(\mathbb{T}^{3}, \mathbb{Z}\right) \cong \mathbb{Z}^{3}$. Количество торов $N_{l} \subset \mathbb{T}^{3}$ должно быть четным, поскольку сумма их гомологических классов равно гомологическому классу поверхности Ферми, которьй равен нулю. (Заметим, что любое гомологически нетривиальное несамопересекающееся двумерное подмногообразие $M \subset \mathbb{T}^{3}$ всегда представляет некоторый неделимый класс гомологий. Любые два 
такие подмногообразия, имеющие пустое пересечение, задают один и тот же с точностью до знака класс гомологий.) Класс $\mu \in H_{2}\left(\mathbb{T}^{3}, \mathbb{Z}\right) \cong \mathbb{Z}^{3}$ определяется тремя взаимно простьми цельми числами: $\mu(B)=\left(m_{1}, m_{2}, m_{3}\right)$.

Целочисленный вектор $\mu(B)$ не изменяется при малых возмущениях вектора $B$. Следовательно, на сфере $S^{2}$ имеется открытое подмножество точек $B$ с одним и тем же $\mu(B)$. Это подмножество, равно как и сам целочисленньй вектор $\mu$, является наблюдаемой характеристикой нашей системы и может быть найдено экспериментально посредством измерения тензора проводимости в присутствии сильного магнитного поля, имеющего направление общего положения. Устойчивое топологически вполне интегрируемое поведение имеет место для всех направлений $B /|B| \in S^{2}$ магнитного поля из открытого всюду плотного подмножества сфферы $S^{2}$. В [11], [12] доказано, что топологическая вполне интегрируемость может не выполняться для направлений $B /|B| \in S^{2}$ магнитного поля из непустого подмножества коразмерности не меньше единицы.

Выражаясь в терминах квазипериодических функций, можно сказать, что “нетипичные” функции $\varphi_{a}(p)=\left.\epsilon(p)\right|_{\mathbb{R}_{B, a}^{2}}$ с тремя квазипериодами, т.е. такие, что открытые компоненты их линий уровня не имеют сильного асимптотического направления, все содержатся в некотором подмножестве, имеющем коразмерность один (в некотором естественном смысле). В [11] построены примеры линий уровня с хаотическим поведением, для которых група частот, изоморфная $\mathbb{Z}^{3}$, всюду плотна в $\mathbb{R}^{2}$. Мы называем такие линии уровня сильно хаотичнымм траекториями. Для таких траекторий были сделаны интересные попытки найти соответствуюшие им свойства физической проводимости. А. Я. Мальцев выдвинул следующую гипотезу [28].

ГиПОТЕЗА 1. Вклад сильно хаотичной траектории в тензор проводимости стремится к нулю при росте $|B|$ (при условии, что $|B|$ остается в разумных пределах), включая проводимость в направлении самого магнитного поля.

Ранее С.П. Царев построил пример “слабо хаотичных” траекторий (неопубликовано, см. [11]). В его случае имеется рациональная линейная зависимость между компонентами поля $B$, поэтому группа частот не всюду плотна в $\mathbb{R}^{2}$. Носитель открытых траекторий всего один, и он совпадает со всей поверхностью Ферми. Однако замькание любой тректории в $\mathbb{T}^{3}$ есть не вся поверхность, а только ее половина, гомеоморфная двумерному тору с двумя дырками. Границы дырок гомологически нетривиальны в $\mathbb{T}^{3}$, поэтому они перестают быть замкнутыми в $\mathbb{R}^{3}$. В примере Царева незамкнутые линии уровня все же имеют асимптотическое направление, но в более слабом смысле:

$$
\gamma(t)=\left(x_{0}, y_{0}\right)+t \cdot\left(x_{1}, y_{1}\right)+o(t),
$$

а проекщия любой траектории на прямую, перпендикулярную вектору $\left(x_{1}, y_{1}\right)$, неограничена.

Интересно взглянуть на поведение траекторий в специальном случае (необшего положения) поверхности Ферми

$$
\epsilon(p)=\cos \left(p_{1}\right)+\cos \left(p_{2}\right)+\cos \left(p_{3}\right)=0 .
$$

В [11], [29] такого рода примеры изучались численно и аналитически. Хаотические траектории появляются для множества магнитных полей, хаусдорфова размерность которого предположительно равна некоторому $\alpha$ из следуюшего промежутка:

$$
1<\alpha<2 \text {. }
$$


На сфрере $S^{2}$ имеется много (даже бесконечно много) зон устойчивой топологической полной интегрируемости, имеющих различные целочисленные характеристики $\mu(B) \in \mathbb{Z}^{3}$. Такого типа примеры мы назьваем сим.метрическими уровнями общего положения, см. ниже.

Мы формулируем следующую гипотезу.

ГиПоТЕЗА 2. (i) Для гомологически тривиальной гладкой поверхности $M_{F} \subset \mathbb{T}^{3}$ общего положения множество направлений магнитного поля, дающих хаотические траектории, имеет в $S^{2}$ хаусдорфову размерность меньше единицы.

(ii) Для однопараметрического семейства $M_{F, t} \subset \mathbb{T}^{3}$ поверхностей Ферми общего положения это множество имеет хаусдорфову размерность меньше двух.

В [11], [12] проведено подробное изучение этой задачи и даны доказательства всех топологических утверждений, необходимых для физических приложений, найденных в [7], [8]. Особое внимание там уделено однопараметрическим семействам поверхностей Ферми, являюшихся уровнями одной и той же функции Морса $f: \mathbb{T}^{3} \rightarrow \mathbb{R}$ :

$$
M_{c}=\{f(p)=c\}
$$

Доказано, что для любого $B$ из зоны устойчивости открытые траектории появляются на уровнях $M_{c}$, образуюших связньй отрезок $c_{1}(B) \leqslant c \leqslant c_{2}(B)$ вешественной прямой. Для $B$ вне всех зон устойчивости сильно хаотичные траектории появляются ровно на одном уровне $c(B) \in \mathbb{R}$. Как следствие, мы получаем следующий результат:

Для функции $f=\epsilon(p)$ с симметрией $\epsilon\left(p+p_{0}\right)=-\epsilon(p)$, где $p_{0} \in \mathbb{T}^{3}$ - некоторый сдвиг, сильно хаотичные траектории не могут появляться на уровнях $M_{c}$ при $c \neq 0$, так как в противном случае они должны также появиться на уровне $M_{-c}$ для того же поля $B$, что невозможно. Уровень $c=0$ такой функции мы назьваем симметричным уровнем общего положения.

Согласно нашей гипотезе, хаусдорфова размерность множества направлений $B /|B| \in S^{2}$, для которых появляются сильно хаотичные траектории, равна некотоpому $\alpha<2$.

Поверхность $\sum_{j=1}^{3} \cos \left(p_{j}\right)=0$ является примером такого симметричного уровня общего положения. Для нее $p_{0}=(\pi, \pi, \pi)$.

Случай трех квазипериодов будет разобран ниже более подробно. Это понадобится нам для доказательства нашего основного результата о квазипериодических функциях с четырьмя квазипериодами (см. следующий раздел).

\section{4. Устойчивая топологическая полная интегрируемость для $n=4$ квазипериодов}

Рассмотрим теперь случай четырех (или более) квазипериодов. Для каждого направления П двумерных плоскостей в $\mathbb{R}^{n}$ (т.е. для каждого двумерного линейного подпространства $\Pi \subset \mathbb{R}^{n}$ ) первоначальная $n$-периодическая функция Морса $f: \mathbb{T}^{n} \rightarrow \mathbb{R}$ задает семейство потомков $\left\{\varphi_{a}(y)\right\}$ на семействе аффинных плоскостей $\mathbb{R}_{\Pi, a}^{2} \subset \mathbb{R}^{n}$, имеющих направление П.

Мыназьваем уровень $\{f=c\}$ функции $f$ топологически вполне интегрируемьм для направления П, если для каждого $\varphi_{a}$ из соответствуюшего семейства потомков 
все регулярные связные компоненты линий уровня $\varphi_{a}(y)=c$ либо компактны, либо имеют сильное асимптотическое направление. Уровень назьвается устойчивым топологически вполне интегрируемым, если это свойство сохраняется при малых возмущениях функции $f$ и направления П, являюшегося точкой многообразия Грассмана $G_{n, 2}$.

Мы говорим, что устойчивьй топологически вполне интегрируемый уровень удовлетворяет условию топологического резонанса (для данного П), если сушествует целочисленная гиперплоскость $\mu \subset \mathbb{R}^{n}, \mu \cap \mathbb{Z}^{n} \cong \mathbb{Z}^{n-1}$, такая, что все открытые регулярные траектории имеют одно и то же асимптотическое направление $\eta_{\Pi \text { П }}$, соппдающее с направлением линии пересечения $\mu \cap \Pi \cong \mathbb{R}$. Так как гиперплоскость $\mu$ целочисленная, она должна оставаться неизменной при малых возмушениях любых исходных объектов.

Отметим, что даже “тривиальньй” случай $n=2$ имеет здесь смысл (как объект элементарной теории Морса на двумерном торе): для дважды периодической функции обшего положения на плоскости существует уровень $f=c$ с одной из связных компонент, представляющей нетривиальньй неделимьй класс гомологий $\mu \in H_{1}\left(\mathbb{T}^{2}, \mathbb{Z}\right)$. Все остальные компоненты любой линии уровня либо гомологически тривиальны, либо гомологичны $\pm \mu$. Для функции Морса с ровно четырьмя критическими точками и попарно различньми критическими значениями $c_{0}<c_{1}<c_{2}<c_{3}$ все уровни $f=c$ при $c_{1}<c<c_{2}$ имеют ровно две связные компоненты, и гомологический класс каждой из них равен $\pm \mu$. Все остальные неособые уровни либо пусты, либо связны и гомологически тривиальны.

Вопрос. Рассмотрим знаменитые вещественные неособые квазипериодические конечнозонные решения уравнения Кд $\Phi$

$$
u(x, t)=2 \partial_{x}^{2} \log \Theta\left(x U+t V+U_{0}\right)+c_{\Gamma}
$$

с произвольным числом квазипериодов (или лакун). Всегда ли уровни $u(x, t)=c$ топологически вполне интегрируемы или они могут быть хаотическими? Как найти их сильное асимптотическое направление и соответствующую целочисленную характеристику $\mu$ ?

П. Г. Гриневич обратил наше внимание на то, что для вещественных конечнозонных решений уравнения Кд $\Phi$-периодическая функция $f=2 \partial_{U}^{2} \log \Theta\left(\eta_{1}, \ldots, \eta_{n}\right)+c_{\Gamma}$ на $\eta$-пространстве всегда является функцией Морса на вешественном $n$-мерном торе с $2^{n}$ критическими точками, попросту потому, что ее можно представить в виде $f=\sum_{j=1}^{n} \alpha_{j} \sin \left(x_{j}\right)$ с помошю некоторого диффеоморфизма тора, изотопного тождественному. В $\eta$-пространстве имеется каноническая решетка, порожденная так называемыми $a$-циклами, которые суть вещественные лакуны спектра одномерного оператора Шрёдингера на гиперэллиптической “спектральной” римановой поверхности $Г$. Вешественные константы $\alpha_{j}$ зависят от спектра.

В настояший момент поведение таких конечнозонных решений изучается численно.

Сосредоточимся теперь на случае $n=4$ квазипериодов. В работе [10] указана идея доказательства того, что для функции Морса $f$ общего положения и некритического уровня $f=c$ обшего положения сушествует открытое всюду плотное множество направлений плоскостей П $\in G_{4,2}$, для которых уровень $f=c$ является устойчивым топологически вполне интегрируемым. Доказательство этого утверждения требует 
развития некоторых результатов работы [12]. Здесь мы сделаем необходимое уточнение утверждения работы [10] и приведем полное доказательство.

Мы полагаем, что уровень общего положения является устойчивым топологически вполне интегрируемым для всех направлений П из некоторого подмножества $S \subset G_{4,2}$ полной меры в $G_{4,2}$. Однако у нас нет соображений, как это можно было бы доказать. Мы не ожидаем никаких подобных явлений при $n>4$.

Теперь мы начнем подробное изучения случая $n=4$. В этом случае даже наш вопрос 1 из предыдущего раздела представляет определенную трудность. Вполне возможно, что на одном уровне квазипериодической функции с четырьмя квазипериодами имеется семейство компактных компонент, размер которых не ограничен сверху никакой константой. Легко построить соответствуюший пример.

Тем не менее, мы собираемся показать, что имеется открытое всюду плотное множество квазипериодических функций $\varphi$ с четырьмя квазипериодами таких, что линии уровня $\varphi=$ const имеют такое же качественное поведение, как и в случае трех квазипериодов. Точная формулировка результата такова.

TеОрема 1. Существуют открытое всюду плотное подмножсество $S \subset$ $C^{\infty}\left(\mathbb{T}^{4}\right)$ 4-периодических функиий $f$ и открытое всюду плотное подмножество $X_{f} \subset G_{4,2}$, зависящее от $f$, такие, что для любого П $\in X_{f}$ любой уровень $M_{c}^{3}=\{f=c\}$ функиии $f$ является устойчивым топологически вполне интегрируемым (либо вообще не содерэст открытых траекторий).

Кроме того, для любой регулярной открытой траектории остаточный член $O(1)$ в (2), а также диаметр любой компактной траектории ограничен сверху константой $C$, которая не зависит от выбора аффинной плоскости $\mathbb{R}_{\Pi, a}^{2}$, содержащей траекторию, при условии, что уровень с и направление $\Pi \in X_{f}$ фиксировань.

Сделаем замечание по поводу обозначений и терминологии. Как только мы перешли от трех квазипериодов к четырем, наша задача перестала быть связанной с физической моделью проводимости в нормальных металлах в присутствии магнитного поля. Поэтому мы изменяем обозначение для координат в пространстве $\mathbb{R}^{n}$ с $p_{l}$, которые использовались для квазиимпульса, на более привычные, $x_{l}, l=0,1,2,3$, и больше не рассматриваем решетку $\mathbb{Z}^{4} \subset \mathbb{R}^{4}$ как двойственную к некоторой физической решетке. "Магнитное поле" $B$ для нас теперь будет отображением из $\mathbb{R}^{4}$ в $\mathbb{R}^{2}$ (или из $\mathbb{R}^{3}$ в $\mathbb{R}$ в случае $n=3$ ) таким, что $\Pi=\operatorname{ker}(B)$. Таким образом, $\mathbb{R}_{\Pi, a}^{2}$ будет обозначать плоскость $B^{-1}(a)$, где $a \in \mathbb{R}^{2}$. В случае $n=3$ мы иногда будем также рассматривать $B$ как вектор, перпендикулярный плоскости П. Тем не менее, мы будем продолжать называть связные компоненты пересечений гиперповерхности $M_{c}^{3}$ с плоскостями $\mathbb{R}_{П, a}^{2}$ траекториями, просто для краткости.

Начнем с того, что сформулируем результаты работ [11], [12] для трехмерного случая в том виде, в котором они понадобятся в дальнейшем. Пусть $B: \mathbb{R}^{3} \rightarrow \mathbb{R}$ линейная функция степени иррациональности три, т.е. функция вида $B(x)=B_{1} x_{1}+$ $B_{2} x_{2}+B_{3} x_{3}$, где вешественные числа $B_{1}, B_{2}, B_{3}$ линейно независимы над $\mathbb{Z}$, и пусть $f: \mathbb{T}^{3} \rightarrow \mathbb{R}$ - гладкая функция обшего положения. Под “функцией обшего положения" мы подразумеваем такую, которая не удовлетворяет некоторым специальным условиям, имеющим коразмерность $\geqslant 1$. Однако мы должны будем уделить внимание особенностям коразмерности один, так как для работы с четырехмерньм случаем нам придется рассматривать однопараметрические семейства трехмерных систем. 
Мы обозначаем одной и той же буквой $f$ функцию на торе и ее поднятие на накрьвающее трехмерное пространство $\mathbb{R}^{3}$. Поверхность уровня $f^{-1}(c)$ в $\mathbb{T}^{3}$ мы обозначаем через $M_{c}^{2}$, а ее накрьвающую в $\mathbb{R}^{3}$ - через $\widehat{M_{c}^{2}}$. Через $\gamma_{a, c}$ обозначается пересечение поверхности $\widehat{M_{c}^{2}}$ с плоскостью $\mathbb{R}_{\Pi, a}^{2}=B^{-1}(a)$. Таким образом, изучаемые нами траектории - это связные компоненты кривой $\gamma_{a, c}$ или ее проекции в $\mathbb{T}^{3}$.

Для начала рассмотрим замкнутые траектории на $M_{c}^{2}$. Заметим, что степень иррациональности вектора $B$ предполагается максимальной, поэтому траектория замкнута в $\mathbb{R}^{3}$ тогда и только тогда, когда замкнут ее образ при проекции в $\mathbb{T}^{3}$. Без предположения о степени иррациональности это может быть неверно, так как тогда замкнутая траектория в $\mathbb{T}^{3}$ может быть негомологична нулю и ее накрьвающая в $\mathbb{R}^{3}$ будет состоять из “периодических" траекторий, которые мы относим к открытым.

Для функции $f$ общего положения на каждом уровне $M_{c}^{2}$ компактные траектории образуют несколько цилиндров, основаниями которых служат либо сепаратрисные циклы, либо точки экстремума ограничения “функщии высоты” $\left.B\right|_{M_{c}^{2}}$. Очевидно, что линейные размеры компактных траекторий (в $\mathbb{R}^{3}$ ) ограничены сверху некоторой константой.

Пусть $U$ - множество таких $c$, что $\gamma_{a, c}$ имеет неограниченные компоненты хотя бы для одного $a$. Иначе говоря, $c$ лежит в $U$ тогда и только тогда, когда $M_{c}^{2}$ содержит открытые регулярные траектории. Следуюшие утверждения, отчасти представленные вьше, можно извлечь из работы [12]:

Множество $U$ либо является замкнутым отрезком, $U=\left[c_{-}, c_{+}\right]$, либо состоит из всего одной точки, $U=\left\{c_{0}\right\}$.

Если $U=\left[c_{-}, c_{+}\right]-$нетривиальный отрезок, то для любого $c \in U$ имеется (единственное) семейство двумерных торов $\mathbb{T}_{c, 1}^{2}, \ldots, \mathbb{T}_{c, 2 k}^{2}$ (где $k$ зависит от $c$ ), вложенных в $\mathbb{T}^{3}$, такое, что

(1) каждый тор $\mathbb{T}_{c, i}^{2}$ состоит из замыкания некоторой открытой траектории в $M_{c}^{2}$ и, возможно, нескольких плоских дисков, перпендикулярных вектоpy $B$;

(2) каждая открытая траектория целиком содержится в одном из торов $\mathbb{T}_{c, i}^{2}$;

(3) все торы $\mathbb{T}_{c, i}^{2}$ задают один и тот же с точностью до знака ненулевой гомологический класс $\mu$ в $H_{2}\left(\mathbb{T}^{3}, \mathbb{Z}\right)$;

(4) для всех, кроме конечного числа, значений $c$ торы $\mathbb{T}_{c, i}^{2}$ попарно не пересекаются, а достаточно малое возмущение уровня $c$ вызывает малую деформацию торов; для исключительных значений $c$ пересечения торов можно устранить малым их шевелением; при таких $c$ пара торов рождается или исчезает.

В этом случае вся картина является устойчивой, т.е. после достаточно малого возмушения $f$ отрезок $U$ и семейство торов $\mathbb{T}_{c, i}^{2}$ также подвергаются малому изменению. Отсюда, в частности, следует, что класс гомологий $\mu$ при таких деформациях не изменяется.

ЗАмЕчАниЕ 1. В построениях работ [11], [12] функция $f$ всегда считалась фиксированной и основным предметом рассмотрения была зависимость поведения нашей динамической системы от магнитного поля $B$ и уровня функции $f$. Устойчивость топологической структуры при малых возмушениях функции $f$ не обсуждалась. Однако аргументы этих работ можно легко усовершенствовать для доказательства такой 
устойчивости. Действительно, ключевым наблюдением в [11], [12] было то, что локально качественное поведение траекторий (включая существование сильного асимптотического направления) зависит только от конечного числа параметров, которые суть некоторые критические значения “функции высоты” $B(x)$, ограниченной на поверхность $\{f=\mathrm{const}\}$. (Скажем, сушествование сильно хаотичных примеров доказано в [11] с помощью указания комбинаторной структуры поверхности и подбора значений параметров.) Нетрудно видеть, что эти параметры “достаточно хорошо” ведут себя при малых изменениях функции $f$, поэтому обобшение вьводов работ [11], [12] на этот, более обший, случай возмущений не требует никаких дополнительных усилий.

Опишем подробнее трехмерную картину. Для поверхности уровня $M^{2} \subset \mathbb{T}^{3}$ обшего положения структура траекторий на $M^{2}$ вьглядит так. Компактные траектории образуют несколько цилиндров, основаниями которых являются экстремумы функции $\left.B\right|_{M^{2}}$ или сепаратрисные циклы, см. рис. 1. Оставшаяся часть поверхности (если она не пуста) состоит из четного числа двумерных торов с дырками или без, причем каждая дырка является сепаратрисным циклом. K каждому такому циклу можно приклеить плоский диск, перпендикулярньй вектору $B$. Обозначим полученную поверхность через $N$. Прообраз $\widehat{N} \subset \mathbb{R}^{3}$ поверхности $N$ относительно стандартной проекции $\nu: \mathbb{R}^{3} \rightarrow \mathbb{T}^{3}$ представляет собой семейство конечньм образом деформированных “гофрированных" плоскостей в $\mathbb{R}^{3}$, см. рис. 2 .
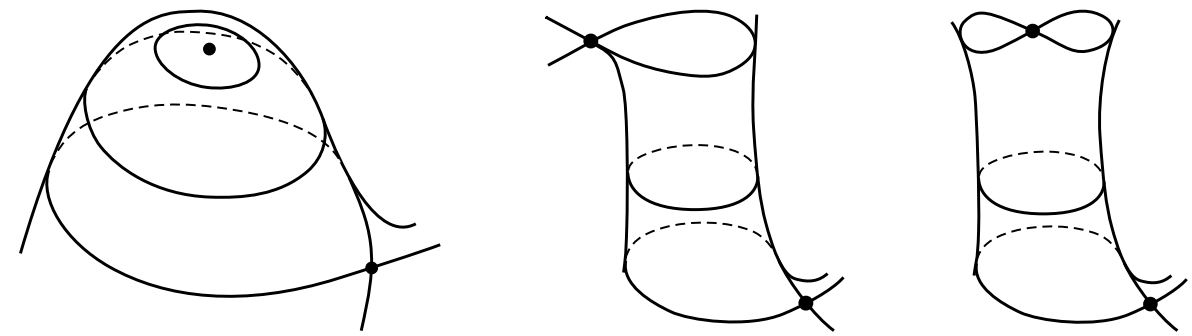

Рис. 1. Цилиндры компактных траекторий

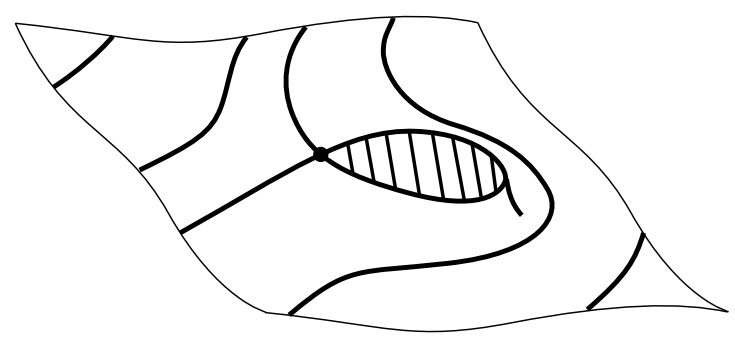

Рис. 2. Гофриированная плоскость

Что происходит с $\widehat{N}$, когда поверхность $M^{2}$ изменяется? Малые деформации поверхности $M^{2}$ приводят лишш к мальм деформациям торов и их накрывающих плоскостей. Предположим, что нам дано однопараметрическое семейство общего положения поверхностей $M^{2}(t)$. Это означает, что мы рассматриваем однопараметрическое семейство общего положения функций $f_{t}: \mathbb{T}^{3} \rightarrow \mathbb{R}$ и для каждого $t$ берем в качестве $M^{2}(t)$ поверхность, заданную уравнением $f_{t}(x)=0$. 
При изменении параметра $t$ связные компоненты поверхности $N$ претерпевают лиш деформации до тех пор, пока они остаются отделенными друг от друга. Однако в процессе деформации может случиться, что два тора сталкиваются и исчезают, или, наоборот, рождаются вновь. Это происходит тогда, когда поверхность $M^{2}(t)$ (как точка бесконечномерного функционального пространства) проходит сквозь подмножество, имеюшее коразмерность один в некотором естественном смысле. При этом неважно, является ли $M^{2}(t)$ семейством поверхностей уровня одной и той же функции или произвольным однопараметрическим семейством поверхностей.

Столкновение торов в случае общего положения описано в [12]. В [12] предполагается, что $M^{2}(t)$ есть семейство поверхностей некоторой функции, $M^{2}(t)=\left\{x \in \mathbb{T}^{3}\right.$ $f(x)=t\}$, однако для произвольного семейства общего положения рассуждение не меняется.

В случае общего положения возможны следующие два вида особенностей, приводящих к рождению или уничтожению пары торов.

(1) Цилиндр замкнутых траекторий с основаниями, прикрепленными к двум различньм компонентам поверхности $N$, схлопьвается. Соответствующее условие коразмерности один имеет следуюший вид: два различных седла соединены сепаратрисой. Это вызывает “взаимодействие” пар открытых траекторий, лежаших на сталкиваюшихся торах, в результате каждая такая пара распадается на бесконечное число замкнутых траекторий, см. рис. 3.

(2) Поверхность $M^{2}$ претерпевает морсовскую перестройку индекса один или два, в результате чего к ней добавляется ручка. Поведение траекторий вблизи особенности показано на рис. 4.

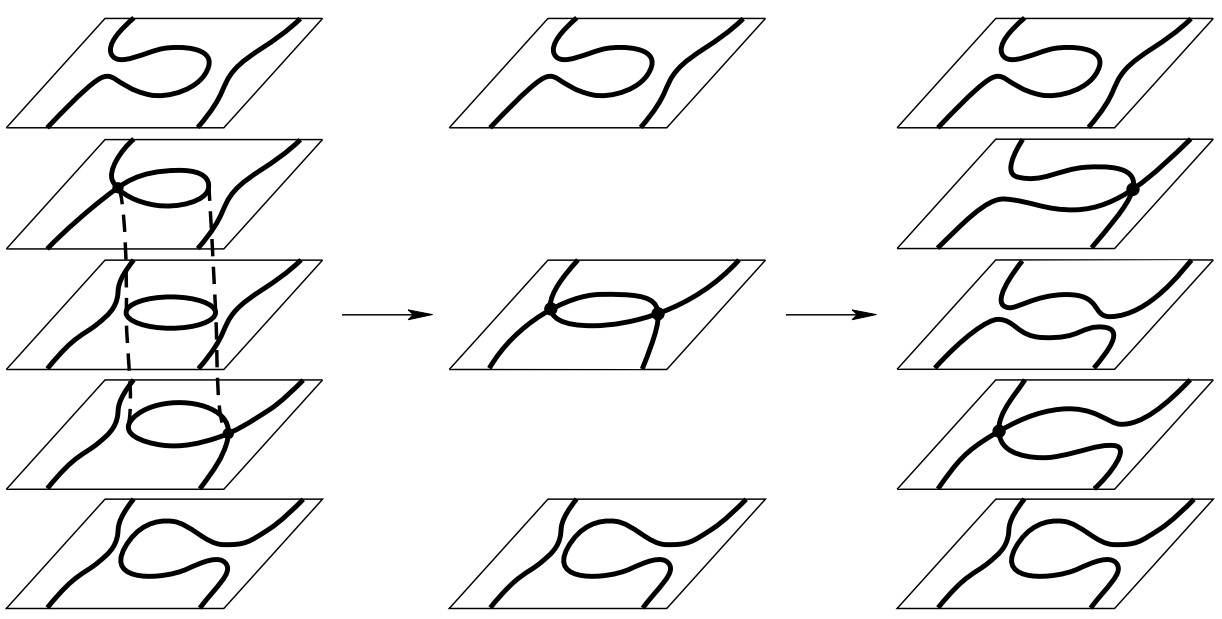

Рис. 3. Схлопьвание цилиндра

Здесь важно отметить, что если $N$ состоит всего из двух торов и $M^{2}(t)$ проходит через особенность одного из двух указанных типов, то все открытые траектории разрушаются и $N$ становится пустым сразу после прохождения особенности. Тем не менее, не доказано, что следующая ситуация невозможна для однопараметрического семейства плоскостей обшего положения: рождение и уничтожение пары торов происходит поочередно в моменты $t_{1}, t_{2}, t_{3}, \ldots$, причем последовательность $\left(t_{n}\right)$ сходится 

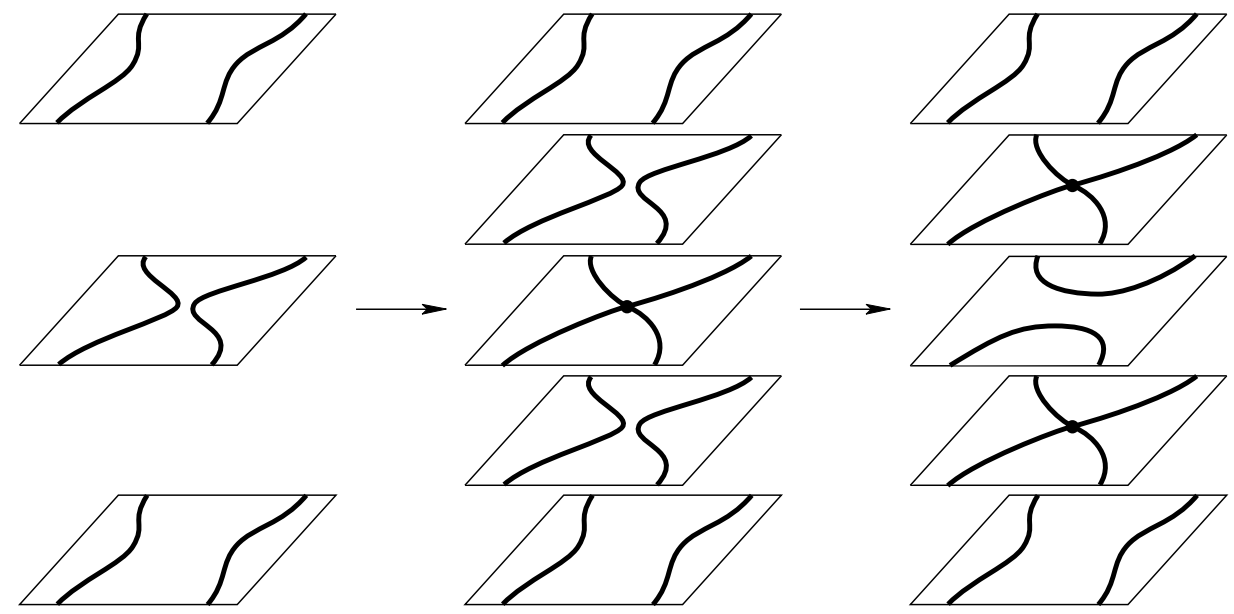

Рис. 4. Морсовская перестройка, разрушающая открытые траектории

к некоторому $t_{*}$, а на $M^{2}\left(t_{*}\right)$ система становится хаотической. Последнее означает, что на поверхности $M^{2}\left(t_{*}\right)$ имеется открытая траектория, замыкание которой имеет род больше единицы (можно показать, что в этом случае он должен быть равен трем). В [11], [12] было доказано существование таких хаотических систем, но показано лишш, что такие системы лежат в подмножестве коразмерности один, что не противоречит их появлению в однопараметрических семействах общего положения. Однако для доказательства нашего основного результата нам не потребуется изучать такие системы.

Обратимся к случаю, в котором $M^{2}(t)=M_{t}^{2}$ является семейством поверхностей уровня одной и той же функции на $\mathbb{T}^{3}$ обшего положения.

Рассмотрим ограничение функции $f$ на плоскость $\mathbb{R}_{\Pi, a}^{2}$ для некоторого $a$. Пусть $V \subset \mathbb{R}_{\Pi, a}^{2}$ - объединение всех компактных компонент множества $\gamma_{a, c}$, взятое по всем $c$, а $W \subset \mathbb{R}_{\Pi, a}^{2}$ - объединение всех неограниченных компонент множества $\gamma_{a, c}$. Будем иметь $\mathbb{R}_{\Pi, a}^{2}=V \cup W, V \cap W=\varnothing, V$ открыто. Пусть $V_{1}, V_{2}, \ldots$ - связные компоненты множества $V$. Заметим, что они не обязательно ограничены. Нетрудно видеть, что функция $f$ постоянна на границе $\partial V_{i}$ каждой компоненты $V_{i}$.

Для $x \in \mathbb{R}_{\Pi, a}^{2}$ положим

$$
\bar{f}(x)= \begin{cases}f(x), & \text { если } x \in W, \\ f\left(\partial V_{i}\right), & \text { если } x \in V_{i}\end{cases}
$$

Сделав так для всех $a$, мы получим новую функцию $\bar{f}: \mathbb{R}^{3} \rightarrow \mathbb{R}$. Мы используем обозначение

$$
N_{c}=\left\{x \in \mathbb{T}^{3} ; \bar{f}(x)=c\right\}
$$

Функция $\bar{f}$ и ее уровни $N_{c}$ имеют следуюшие свойства.

ЛЕмма 1. Функиия $\bar{f}$ корректно определена и непрерывна на торе $\mathbb{T}^{3}=\mathbb{R}^{3} / \mathbb{Z}^{3}$. Eсли $U=\left\{c_{0}\right\}$, mo $\bar{f} \equiv c_{0}$. 
Если $U=\left[c_{-}, c_{+}\right]$, где $c_{-}<c_{+}$, то для всех, кроме конечного числа, $c \in$ $\left(c_{-}, c_{+}\right)$выполнено

$$
N_{c}=\bigcup_{i} \mathbb{T}_{c, i}^{2}
$$

Для всех $c \in\left[c_{-}, c_{+}\right]$малая регулярная окрестность мнохсества $N_{c}$ гомеоморфна обгединению нескольких әкземпляров многообразия $\mathbb{T}^{2} \times[0,1]$.

ДокАЗАТЕЛЬСтво. В случае $U=\left\{c_{0}\right\}$ наше утверждение тривиально.

Предположим, что $U=\left[c_{-}, c_{+}\right]$, причем $c_{-}<c_{+}$. По построению $\mathbb{T}_{c, i}^{2} \cap M_{c}$ состоит из открытых траекторий, поэтому $\bar{f}(x) \equiv c$ на $\mathbb{T}_{c, i}^{2} \cap M_{c}$. Весь тор $\mathbb{T}_{c, i}^{2}$ получается из $\mathbb{T}_{c, i}^{2} \cap M_{c}$ добавлением дисков, каждый из которых лежит в плоскости $\mathbb{R}_{\Pi, a}^{2}$ для некоторого $a$. Край такого диска является частью особой неограниченной связной компоненты линии уровня функции $\left.f\right|_{\mathbb{R}_{\Pi, a}^{2}}$. По построению внутри такого диска мы имеем $\bar{f} \equiv c$. Следовательно, $\mathbb{T}_{c, i}^{2} \subset N_{c}$.

Изучим, что происходит с торами $\mathbb{T}_{c, i}^{2}$ при изменении $c$. Для всех, кроме конечного числа, значений $c$ торы $\mathbb{T}_{c, i}^{2}, \mathbb{T}_{c, j}^{2}$ попарно не пересекаются при $i \neq j$. Кроме того, если $c \neq c^{\prime}$, то торы $\mathbb{T}_{c, i}^{2}$ и $\mathbb{T}_{c^{\prime}, j}^{2}$ не пересекаются для любых $i, j$.

Когда $c$ изменяется, торы $\mathbb{T}_{c, i}^{2}$ непрерьвно деформируются, за исключением нескольких значений $c$, при которых происходит одно из следующих двух событий: 1) два тора сталкиваются и исчезают; 2) два тора рождаются. Второе событие обратно первому.

Опишем столкновение торов более подробно. В момент столкновения в $\mathbb{T}^{3}$ имеется замкнутое подмножество $W$, представляющее собой многообразие $\mathbb{T}^{2} \times[0,1]$, в котором некоторые отрезки $x \times[0,1]$ сжаты в точку. Такая точка $x \in \mathbb{T}^{2}$ может быть ровно одна, а может быть цельй замкнутьй диск $D^{2} \subset \mathbb{T}^{2}$ таких точек. Первьй случай соответствует морсовской перестройке поверхности уровня функции $f$, а второйсхлопьванию цилиндра замкнутых траекторий.

Внутренность множества $W$ состоит из замкнутых траекторий, и по построению функция $\bar{f}$ постоянна на всем $W$. Таким образом, $W$ есть связная компонента некоторого $N_{c}$, поскольку $W$ зажато между двумя столкнувшимися торами. Мы называем такое $W$ псевдотором.

Таким образом, мы имеем следуюшее. Разложение тора $\mathbb{T}^{3}$ в объединение связных компонент уровней $N_{c}$ представляет собой не что иное, как тривиальное расслоение над окружностью $S^{1}$ со слоем $\mathbb{T}^{2}$, в котором некоторые слои заменены на псевдоторы. Это показано схематически на рис. 5.

Вернемся к четырехмерному случаю. Пусть $\Pi \in G_{4,2}$ - двумерная плоскость, заданная как ядро линейного отображения $B: \mathbb{R}^{4} \rightarrow \mathbb{R}^{2}$. Через $\mathbb{R}_{\Pi, a, b}^{2}$ мы обозначаем афффинную плоскость $B^{-1}(a, b) \subset \mathbb{R}^{4}$, а через $M_{\leqslant c}^{4}\left(M_{\geqslant c}^{4}\right)$ - подмножество тора $\mathbb{T}^{4}$, заданное неравенством $f(x) \leqslant c$ (соответственно $f(x) \geqslant c)$.

Пусть $N \subset \mathbb{T}^{4}$ - некоторое подмногообразие или, более общо, некоторое подмножество. Скажем, что $N$ лежит преимущественно ниже (преимущественно выше) уровня $M_{c}^{3}$, если для любых $a, b \in \mathbb{R}$ пересечение $\widehat{N} \cap \mathbb{R}_{\Pi, a, b}^{2}$ не пересекается ни с одной неограниченной компонентой множества $\widehat{M}_{\geqslant c}^{4} \cap \mathbb{R}_{\Pi, a, b}^{2}$ (соответственно множества $\left.\widehat{M}_{\leqslant c}^{4} \cap \mathbb{R}_{\Pi, a, b}^{2}\right)$. Таким образом, свойство подмножества $N$ лежать преимущественно ниже $M_{c}^{3}$ зависит от П. 


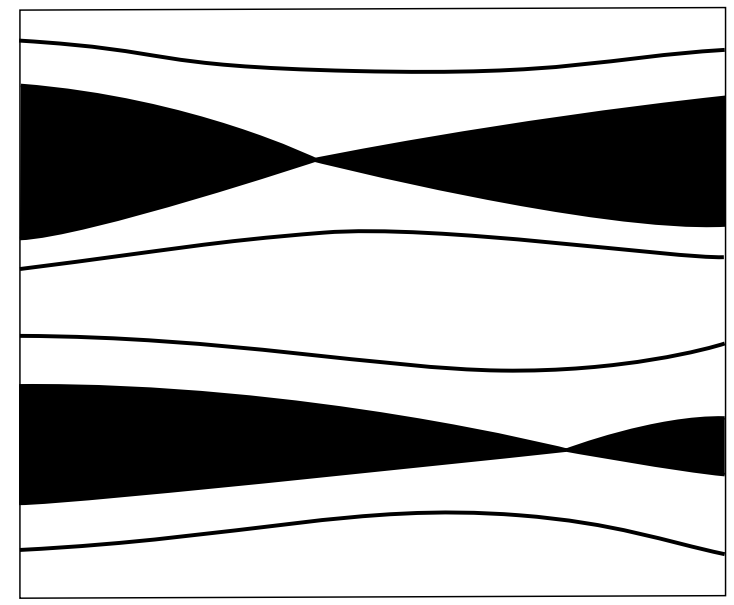

Рис. 5. Семейство торов, некоторые из которых заменены на псевдоторы

Доказательство следуюших двух фактов не содержит ничего нового по сравнению с трехмерным случаем.

ЛЕмма 2. Если $N$ лежит преимущественно ниже или преимущественно выше $M_{c}^{3}$ для данного П, то это свойство сохраняется при малых возмущениях плоскости П, функиии $f$ и значения $c$.

ЛЕмма 3. Если для данных $f$, П и с найдется гомологически нетривиальный трехмерный тор $N$, лехсащий преимущественно выще или преимущественно ниже $M_{c}^{3}$, то для этих $f$, П и с выполнено утвержсдение теоремы 1.

Таким образом, для доказательства теоремы 1 достаточно показать, что для всюду плотного множества пар $(f, \Pi)$ при произвольном $c$ существует гомологически нетривиальный трехмерньй тор $N \subset \mathbb{T}^{4}$, лежаший преимущественно вьше или преимушественно ниже $M_{c}^{3}$.

Пусть $B=\left(\ell_{1}, \ell_{2}\right)$ - пара линейных функций на $\mathbb{R}^{4}$ таких, что

(1) функция $\ell_{1}$ рациональна, т.е. $\ell_{1} \in\left(\mathbb{Z}^{4}\right)^{*}$;

(2) ограничение функции $\ell_{2}$ на целочисленную гиперплоскость $\left\{\ell_{1}=0\right\}$ имеет степень иррациональности три.

Очевидно, что множество двумерных плоскостей $\Pi=\operatorname{ker} B$, заданных такими $\ell_{1}, \ell_{2}$, всюду плотно в $G_{4,2}$.

Не ограничивая обшности, можно считать, что $\ell_{1}(x)=x_{0}, \ell_{2}(x)=H_{1} x_{1}+H_{2} x_{2}+$ $H_{3} x_{3}$, где $x=\left(x_{0}, x_{1}, x_{2}, x_{3}\right) \in \mathbb{R}^{4}, \operatorname{rank}_{\mathbb{Z}}\left\langle H_{1}, H_{2}, H_{3}\right\rangle=3$. Рассмотрим четырехмерньй тор $\mathbb{T}^{4}$ как однопараметрическое семейство трехмерных торов $\mathbb{T}_{t}^{3}=\left\{x_{0}=t\right\}$. Для каждого $t \in[0,1]$ мы рассмотрим ограничения $f_{t}$ и $\ell_{2, t}$ соответственно функций $f$ и $\ell_{2}$ на $\mathbb{T}_{t}^{3}$ так, как в трехмерном случае. Для каждого $t$ определим $U_{t}=\left[c_{t-}, c_{t+}\right]$, $\overline{f_{t}}, N_{t, c}$ как и раньше.

Рассмотрим зависимость отрезка $U_{t}$ от $t$. Коншы $c_{t \pm}$ этого отрезка являются непрерьвньми функциями от $t$. Кроме того, в областях, где $c_{t+}>c_{t-}$, эти функции кусочно-гладкие. Это следует из того, что локально, возле $t$ общего положения, эти функции задаются условиями вида: два седла на $M_{t, c_{ \pm}}^{2}$ соединены сепаратрисой, или 
вида: $c$ является критическим значением функции $f_{t}$, соответствуюшим критической точке индекса 1 или 2. Здесь мы назьваем такие области зонами устойчивости. Каждой зоне устойчивости соответствует целочисленный вектор $\mu \in H_{1}\left(\mathbb{T}^{3}, \mathbb{Z}\right)=\mathbb{Z}^{3}$.
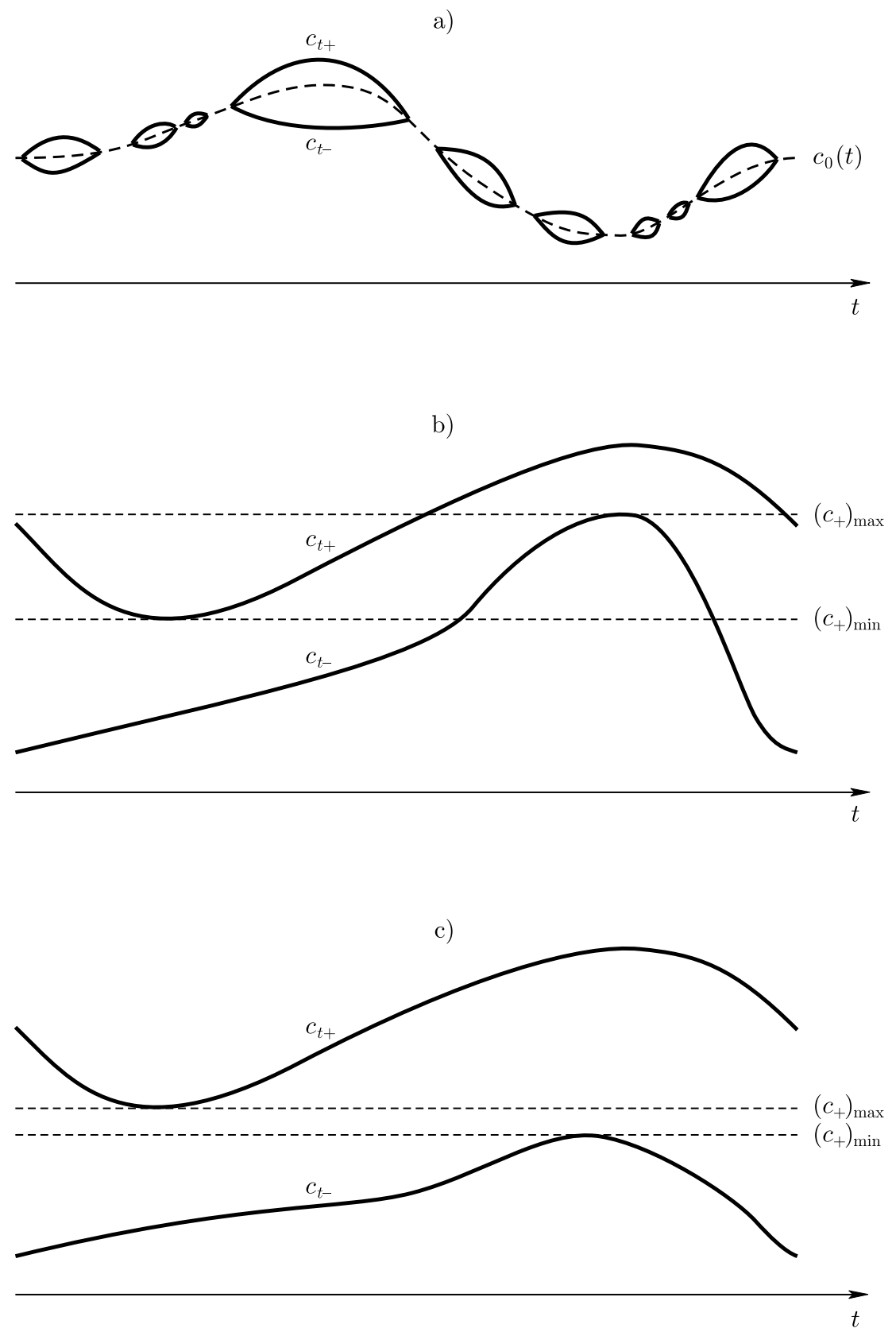

Рис. 6. Функции $c_{t \pm}$ в случае общего положения 
На рис. 6 показано, как функции $c_{t \pm}$ могут себя вести в случае общего положения. Возможно, что при некотором $t$ имеет место равенство $c_{t+}=c_{t-}$, см. рис. $\left.6 \mathrm{a}\right)$. Это может произойти на границе зоны устойчивости или при $t$ таком, что открытые траектории на $M_{t}^{3}$ хаотичны. Для всех таких $t$ мы имеем $c_{t+}=c_{t-}=c_{0}(t)$, где $c_{0}(t)-$ некоторая кусочно-гладкая функция от $t$, локально определяемая условием вида: сумма "высот" некоторых седел равна нулю, см. [11], [12].

По всей видимости, любое "хаотичное" $t$ должно быть точкой накопления бесконечной последовательности зон устойчивости. Иначе говоря, невозможна ситуация, при которой равенство $c_{t+}=c_{t-}$ вьполняется всюду на нетривиальном интервале $\left(t_{1}, t_{2}\right)$. Однако непосредственно из [11], [12] это не следует и в настояшей работе использоваться не будет.

Лемма 4. Для функиии $f$ общего положения равенство

$$
\min _{t} c_{t+}=\max _{t} c_{t-}
$$

не имеет места.

ДокАЗАТЕЛЬСТвО. Нужно рассмотреть следующие три случая.

Случай 1 . Для всех $t$ выполнено $c_{t+}>c_{t-}$. Тогда существует гладкая периодическая функция $g(t)$ такая, что $c_{t+}>g(t)>c_{t-}$. Условие (3) не будет вьполняться, если мы возмутим функцию $f$ следуюшим образом: $f\left(t, x_{1}, x_{2}, x_{3}\right) \mapsto f_{\varepsilon}\left(t, x_{1}, x_{2}, x_{3}\right)=$ $f\left(t, x_{1}, x_{2}, x_{3}\right)+\varepsilon g(t)$, где $|\varepsilon|$ достаточно мало. Таким образом, в этом случае (3) является условием коразмерности один на функцию $f$.

Случай 2. Для двух различных значений $t=t_{1}, t_{2}$ мы имеем $c_{t+}=c_{t-}$. Тогда условие (3) не будет выполняться после достаточно малого возмущения $f \mapsto f+\varepsilon g(t)$, где $g(t)$ - произвольная функция, для которой $g\left(t_{1}\right) \neq g\left(t_{2}\right)$.

Случай 3 . Имеется ровно одно значение $t=t_{0}$, при котором $c_{t+}=c_{t-}=c_{0}$, а для всех остальных $t$ мы имеем $c_{t+}>c>c_{t-}$. Возьмем $t$, близкое к $t_{0}$. Тогда отрезок $\left[c_{t-}, c_{t+}\right]$ будет мал, что означает следуюшее: когда $c$ варьируется от $\left(c_{t-}-\delta\right)$ до $\left(c_{t+}+\delta\right)$, где $\delta>0$, пара торов $N_{t, c}$ рождается в момент $c=c_{t-}$ и в момент $c=c_{t+}$, т.е. почти сразу же, исчезает. Следовательно, на $M_{t, c_{0}}$ имеется пара цилиндров замкнутых траекторий очень малой высоты.

Теперь зафиксируем $c=c_{0}$ и будем варьировать $t$. Когда $t$ приближается к $t_{0}$, скажем, слева, мы будем иметь два цилиндра замкнутых траекторий, которые оба вырождаются в момент $t=t_{0}$. Когда $t$ проходит через $t_{0}$, два цилиндра появляются снова. Ключевую роль здесь играет то, что эти, новые, цилиндры появляются из той же пары вырожденных цилиндров. Действительно, пара вырожденных цилидров, получаемая при подходе к $t_{0}$ слева, разрезает поверхность $M_{c_{0}, t_{0}}$ (из которой удалены все невырожденные цилиндры замкнутых траекторий) на два тора. Можно показать, что так как степень иррациональности функции $\ell_{2}$ равна трем, на $M_{t_{0}, c_{0}}$ не может быть других вырожденных цилиндров.

Таким образом, мы имеем следующую ситуацию. Когда $t$ проходит от $t_{0}-\delta \mathrm{k} t_{0}+\delta$, два цилиндра замкнутых траекторий исчезают и тут же рождаются вновь. Пусть $h_{1}(t), h_{2}(t)$ - их длины в момент времени $t$. Имеют место не только равенства $h_{1}\left(t_{0}\right)=$ $h_{2}\left(t_{0}\right)=0$, но и $h_{1}^{\prime}\left(t_{0}\right)=h_{2}^{\prime}\left(t_{0}\right)=0$, что накладывает ограничение коразмерности два на функцию $f$.

По построению мы имеем следуюшее. 
Лемма 5. Для любого $t$ и любого $c>c_{t+}\left(c<c_{t-}\right)$ mор $\mathbb{T}_{t}^{3}$ лежит преимущественно ниже (соответственно выше) $M_{c}^{3}$.

Согласно лемме 4, возможны только следуюшие два случая:

1) $\min _{t} c_{t+}<\max _{t} c_{t-}$;

2) $\min _{t} c_{t+}>\max _{t} c_{t-}$.

В случае 1) для любого $c$ мы будем иметь либо $c>\min _{t} c_{t+}$, либо $c<\max _{t} c_{t-}$, и по лемме 5 доказательство закончено.

Остается разобрать случай 2$), \min _{t} c_{t+}>\max _{t} c_{t_{-}}$. Это неравенство означает, в частности, что отрезки $U_{t}$ имеют нетривиальное общее пересечение $U=\bigcap_{t} U_{t}=$ $\left[c_{-}, c_{+}\right]$, а значит, зона устойчивости всего одна, и она покрьвает всю окружность $S^{1}$. Этот случай проиллюстрирован на рис. $6 \mathrm{c})$.

Определим функцию $\bar{f}: \mathbb{T}^{4} \rightarrow \mathbb{R}$ так же, как в трехмерном случае, рассмотрев пересечения $M_{c}^{3} \cap \mathbb{R}_{\Pi, a, b}^{2}$, и введем обозначение

$$
N_{c}=\left\{x \in \mathbb{T}^{4} ; \bar{f}(x)=c\right\}
$$

По построению $\overline{f_{t}}$ совпадает с ограничением функщии $\bar{f}$ на $\mathbb{T}_{t}^{3}$, и мы имеем

$$
N_{c}=\bigcup_{t} N_{t, c}
$$

Для почти всех значений $c, t$ пересечение $N_{c} \cap \mathbb{T}_{t}^{3}$ либо пусто, либо состоит из двумерных торов, имеющих с точностью до знака один и тот же класс гомологий $\alpha \in H_{2}\left(\mathbb{T}^{3}, \mathbb{Z}\right)$. В этом случае весь тор $\mathbb{T}^{4}$ оказьвается снабжен структурой тривиального $\mathbb{T}^{2}$-расслоения над $\mathbb{T}^{2}$ с однопараметрическим семейством слоев, замененных на псевдоторы. Функция $\bar{f}$ постоянна на каждом слое, так что ее можно рассматривать как функцию на базе $\mathbb{T}^{2}$ этого расслоения.

На рис. 7 изображена структура линий уровня функции $\bar{f}$, рассматриваемой как функция на $\mathbb{T}^{2}$. Точке общего положения соответствует двумерньй тор, вложенньй в $\mathbb{T}^{4}$, причем все эти двумерные торы “параллельны”. Точкам, где ограничение функции $\bar{f}$ на прямую $t=$ const имеет экстремум, соответствуют псевдоторы.

Включение псевдоторов в однопараметрическое семейство двумерных торов не меняет топологического типа объединения этих торов. Действительно, как было отмечено вьше, малая регулярная окрестность псевдотора в $\mathbb{T}^{3}$ гомеоморфна $\mathbb{T}^{2} \times(0,1)$. Иначе говоря, приклеивание "воротника" $\mathbb{T}^{2} \times(0,1)$ к псевдотору снова дает $\mathbb{T}^{2} \times$ $(0,1)$.

Мы приходим к следующему выводу.

Лемма 6. Для почти всех с каждая связная компонента множества $N_{c}$ гомеоморфна $\mathbb{T}^{3}$. Исключение составляют те $c$, которые являются критическими значениями функиии $\bar{f}$ на $\mathbb{T}^{2}$.

Для функции $f$ общего положения мы получим морсовскую функцию общего положения $\bar{f}$ на $\mathbb{T}^{2}$. Для такой функции должен быть отрезок $\left[c_{1}, c_{2}\right]$ такой, что для любого $c \in\left[c_{1}, c_{2}\right]$ линия уровня $\bar{f}=c$ содержит замкнутую кривую, не гомологичную нулю в $\mathbb{T}^{2}$. Прообраз $N_{c}$ этой линии уровня в $\mathbb{T}^{4}$ будет иметь связную компоненту, гомеоморфную $\mathbb{T}^{3}$ и не гомологичную нулю. Таким образом, мы показали следующее. 


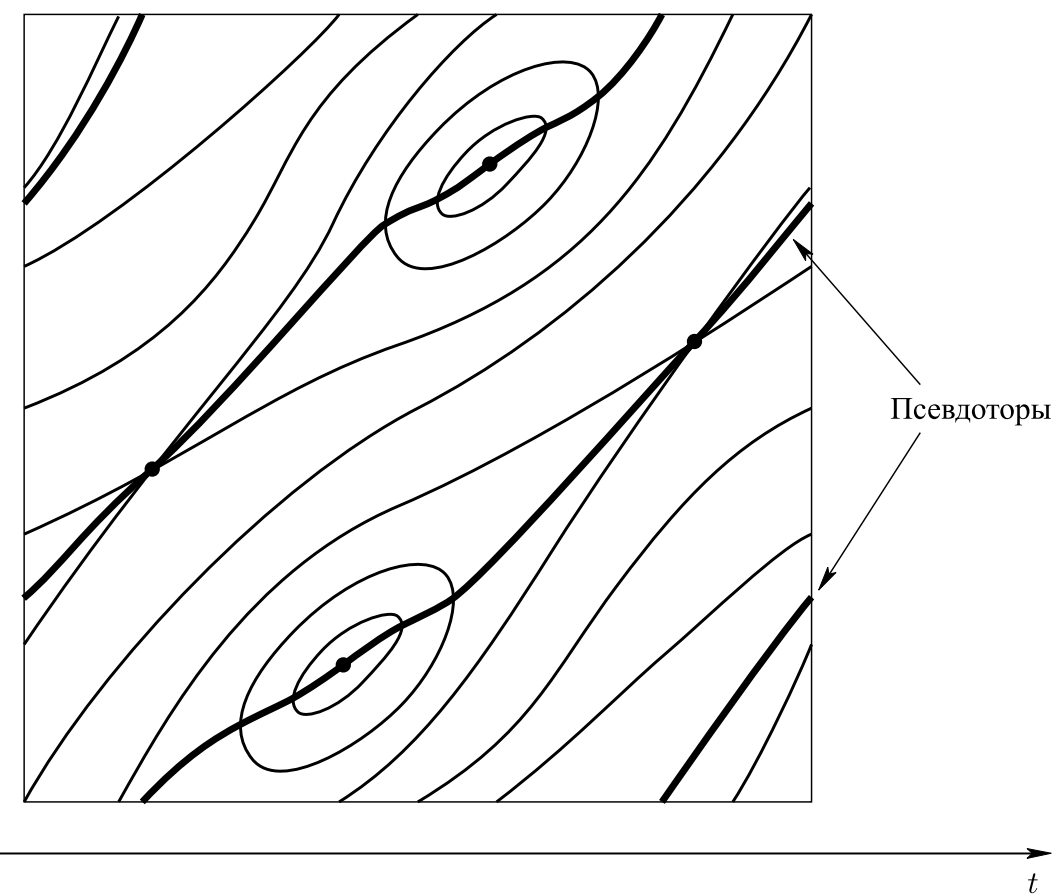

Рис. 7. Линии уровня функции $\bar{f}$ на $\mathbb{T}^{2}$

Лемма 7. Найдутся $c_{1}, c_{2}$ такие, что $c_{1}<c_{2}$ и каждое из $N_{c_{1}}, N_{c_{2}}$ содержит связную компоненту, гомеоморфную $\mathbb{T}^{3}$ и не гомологичную нулю.

Остается заметить, что при $c>c_{1}$ гиперповерхность $N_{c_{1}}$ лежит преимущественно ниже $M_{c}^{3}$, а при $c<c_{2}$ гиперповерхность $N_{c_{2}}$ лежит преимушественно вьше $M_{c}^{3}$. Таким образом, для всех $c$ найдется не гомологичный нулю трехмерньй тор, лежащий либо преимушественно вьше, либо преимушественно ниже $M_{c}^{3}$, что завершает доказательство в случае 2).

\section{СПИСОК ЛИТЕРАТУРЫ}

[1] С. П. Новиков. Гамильтонов формализм и многозначньй аналог теории Морса // УМН. 1982. T. 37. № 5. C. 3-49; http://www.mi.ras.ru/ snovikov/74.zip.

[2] А. В. Зорич. Задача С.П. Новикова о полуклассическом движении электрона в однородном магнитном поле, близком к рациональному // УМН. 1984. Т. 39. № 5. С. 235-236.

[3] S.P. Novikov. Quasiperiodic structures in topology // Topological Methods in Modern Mathematics (Stony Brook, NY, 1991). Houston, TX: Publish or Perish, 1993. P. 223-233.

[4] И. А. Дынников. Доказательство гипотезы С. П. Новикова для случая малшхх возмущений рациональных магнитных полей // УМН. 1992. Т. 47. № 3. С. 161-162.

[5] И.А. Дынников. Задача С.П. Новикова о полуклассическом движении электрона // УМН. 1993. Т. 48. № 2. С. 179-180.

[6] И.А. Дынников. Доказательство гипотезы С. П. Новикова о полуклассическом движении электрона // Матем. заметки. 1993. Т. 53. № 5. С. 57-68.

[7] С. П. Новиков, А.Я. Мальцев. Топологические квантовые характеристики, наблюдаемые при изучении проводимости в нормальных металлах // Письма в ЖЭТФ. 1996. Т. 63. № 10. C. $809-813$. 
[8] С. П. Новиков, А. Я. Мальцев. Топологические явления в нормалњных металлах // УФН. 1998. Т. 41. № 3. С. 231-239; arXiv:cond-mat/9709007.

[9] И. М. Лифшиц, М.Я. Азбель, М. И. Каганов. Электронная теория металлов. М.: Наука, 1971.

[10] С. П. Новиков. Уровни квазипериодических функций на плоскости и гамильтоновы системы // УМН. 1999. Т. 54. № 5. С. 147-148; arXiv:math-ph/9909032.

[11] I. A. Dynnikov. Semiclassical motion of the electron. A proof of the Novikov conjecture in general position and counterexamples // Solitons, Geometry, and Topology: On the Crossroad. Providence, RI: Amer. Math. Soc., 1997. P. 45-73. (Amer. Math. Soc. Transl. Ser. 2. V. 179.)

[12] И.А. Дынников. Геометрия зон устойчивости в задаче С. П. Новикова о полуклассическом движении электрона // УМН. 1999. Т. 54. № 1. С. 21-60.

[13] A. Ya. Maltsev. Quasiperiodic functions theory and the superlattice potentials for a two-dimensional electron gas // J. Math. Phys. 2004. V. 45. № 3. P. 1128-1149; arXiv:cond-mat/0302014.

[14] Т. К. Т. Ле, С. А. Пиунихин, В.А. Садов. Геометрия квазикристаллов // УМН. 1993. T. 48. № 1. C. 41-102.

[15] V.I. Arnold, A.B. Givental. Symplectic geometry // Dynamical Systems. V. IV: Symplectic Geometry and Its Applications / ed. V.I. Arnold, S.P. Novikov. Berlin: Springer-Verlag, 2001. P. 1-38. (Encyclopedia Math. Sci. V. 4.)

[16] B. A. Dubrovin, I. M. Krichever, S. P. Novikov. Integrable systems. I // Dynamical Systems. V. IV: Symplectic Geometry and Its Applications / ed. V. I. Arnold, S.P. Novikov. Berlin: Springer-Verlag, 2001. P. 177-332. (Encyclopedia Math. Sci. V. 4.)

[17] А. А. Абрикосов. Основы теории металлов. М.: Наука, 1987.

[18] C. Kittel. Quantum Theory of Solids. New York: Wiley, 1963.

[19] J. M. Ziman. Principles of the theory of solids. London: Cambridge Univ. Press 1972.

[20] И. М. Лифошиц, В.Г. Песчанский. Галвваномагнитные характеристики металлов с открытыми поверхностями Ферми. II // ЖЭТФ. 1960. Т. 38. С. 188-193.

[21] И.А. Дынников, А.Я. Мальцев. Топологические характеристики электронного спектра в монокристаллах // ЖЭТФ. 1997. Т. 112. № 1. С. 371-378.

[22] A. Ya. Maltsev, S. P. Novikov. Dynamical systems, topology, and conductivity in normal metals // J. Statist. Phys. 2004. V. 115. № 1. P. 31-46; arXiv:cond-mat/0312708.

[23] A. Ya. Maltsev, S. P. Novikov. Quasiperiodic functions and dynamical systems in quantum solid state physics // Bull. Braz. Math. Soc. (N.S.). 2003. V. 34. №1. P. 171-210; arXiv:math-ph/0301033.

[24] A. Ya. Maltsev, S. P. Novikov. Topology, quasiperiodic functions and the transport phenomena // Topology in Condensed Matters / ed. M.I. Monastyrsky. Berlin: Sprinter-Verlag, 2005 (to appear); arXiv:cond-mat/0312710.

[25] C. W. J. Beenakker. Guiding-center-drift resonance in a periodically modulated two-dimensional electron gas // Phys. Rev. Lett. 1989. V. 62. № 17. P. 2020-2023.

[26] В.И. Арнольд. Топологические и эргодические свойства замкнутых 1-форм с несоизмеримыми периодами // Функц. анализ и его прил. 1991. Т. 25. № 2. С. 1-12.

[27] Я.Г. Синай, К. М. Ханин. Перемешивание некоторых классов специальных потоков над поворотом окружности // Функц. анализ и его прил. 1992. Т. 26. № 3. С. 1-21.

[28] А. Я. Мальцев. Аномальное поведение тензора электропроводности в сильных магнитных полях // ЖЭТФ. 1997. Т. 112. № 5 (11). С. 1710-1726.

[29] R. De Leo. Numerical analysis of the Novikov problem of a normal metal in a strong magnetic field // SIAM J. Appl. Dyn. Syst. 2003. V. 2. № 4. P. 517-545.

Московский государственньй университет им. М.В.Ломоносова;

Поступила в редакцию Институт теоретической физики им. Л. Д. Ландау, IPST, 26.12 .2004

University of Maryland at College Park

E-mail: dynnikov@mech.math.msu.su; novikov@glue.umd.edu 